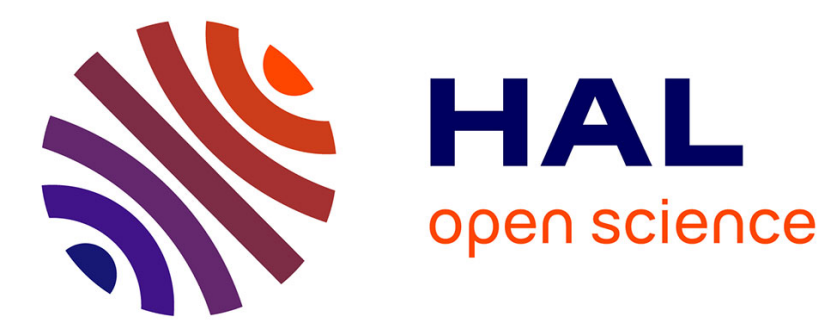

\title{
Hydrogen-bonded diketopyrrolopyrrole derivatives for energy-related applications
}

\author{
Amparo Ruiz-Carretero, Nelson Ricardo Ávila Rovelo, Swann Militzer, \\ Philippe Mesini
}

\section{- To cite this version:}

Amparo Ruiz-Carretero, Nelson Ricardo Ávila Rovelo, Swann Militzer, Philippe Mesini. Hydrogenbonded diketopyrrolopyrrole derivatives for energy-related applications. Journal of Materials Chemistry A, 2019, 7 (41), pp.23451-23475. 10.1039/C9TA05236D . hal-02400909

\section{HAL Id: hal-02400909 \\ https://hal.science/hal-02400909}

Submitted on 8 Jan 2021

HAL is a multi-disciplinary open access archive for the deposit and dissemination of scientific research documents, whether they are published or not. The documents may come from teaching and research institutions in France or abroad, or from public or private research centers.
L'archive ouverte pluridisciplinaire HAL, est destinée au dépôt et à la diffusion de documents scientifiques de niveau recherche, publiés ou non, émanant des établissements d'enseignement et de recherche français ou étrangers, des laboratoires publics ou privés. 


\title{
Hydrogen-bonded diketopyrrolopyrrole derivatives for energy-related applications: optoelectronic and self-assembly properties, morphology and devices
}

\author{
Amparo Ruiz-Carretero, ${ }^{*[a]}$ Ricardo Ávila Rovelo, ${ }^{[a]}$ Swann Militzer, ${ }^{[a]}$ Philippe J. Mésini, ${ }^{[a, b]}$

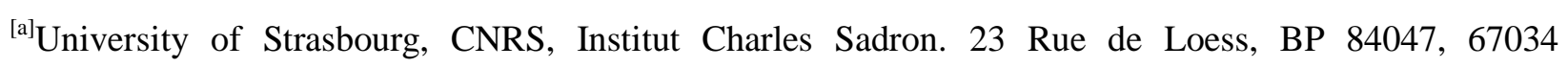 \\ Strasbourg, Cedex 2, France. E-mail: amparo.ruiz@ics-cnrs.unistra.fr \\ ${ }^{[b]}$ International Center for Frontier Research in Chemistry, 8 allée Gaspard Monge, 67000 Strasbourg, \\ France.
}

\begin{abstract}
Since the discovery of the diketopyrrolopyrrole (DPP) dye in the 70's it has been one of the most used pigments in industrial applications and in the search of new materials. They are great electroactive molecules extensively studied in the field of organic electronics thanks to their optoelectronic properties that arise from its conjugated core and the functionalization in the 3- and 6- positions. DPPs properties can be regulated synthetically by introducing different substituents or by tuning the aggregation state using noncovalent interactions, such as hydrogen-bonding in combination with $\pi$ - $\pi$ stacking. This review outlines the advances made in organic electronics using H-bonding functionalization on DPP derivatives. The examples of H-bonded DPPs using the amide groups in their structures and the functionalization with H-bonding motifs in different positions of the molecule will be discussed. The influence of such noncovalent interactions on the optoelectronic properties and the correlation with morphology and the results obtained in different energy related applications will be reviewed.
\end{abstract}

\section{Key words}

Hydrogen-bonding; Diketopyrrolopyrrole; Organic Electronics; Optoelectronic properties; Morphology 


\section{Introduction}

Supramolecular chemistry ${ }^{1}$ uses the power of noncovalent interactions among many individual species to achieve very precise structures with properties and functions not present when such species are isolated. Hydrogen bonds (H-bonds) are one of the noncovalent interactions employed to direct supramolecular assemblies into directional and well-organized structures. ${ }^{2}$ H-bonds are sensitive to temperature, solvent, concentration and chirality among other parameters, being possible to tune the aggregation state by varying any of them. This is very useful to program molecules to form the appropriate structures that will present specific properties and functions. This is the case of hydrogenbonded semiconductors, ${ }^{3}$ where not only the electronic properties play an important role in the final outcome. The molecular packing and aggregation in solution and on thin film are equally important, making possible to influence the optoelectronic properties upon self-assembly. Diketopyrrolopyrrole (DPP) dyes are among the best electroactive segments used in semiconductors, ${ }^{4,5}$ since their discovery by Farnum $^{6}$ in the 70 's while trying to synthesize azetinone, DPP has been one of the most used pigments in industrial applications and in the search of new materials. Multiple examples of polymers and small molecules can be found for the fabrication of organic solar cells, ${ }^{7}$ organic field effect transistors, ${ }^{8,9}$ and sensors. ${ }^{10,11}$ The optoelectronic properties of this molecule arise from its conjugated core and the functionalization in the 3- and 6- positions, being thiophene, phenyl and furan the main aromatic moieties used. Subsequently, further functionalization can be added according to the specific needs and the desired applications. For instance, electron-withdrawing or electron-donating groups can be introduced or the conjugation can be further extended using a push-pull strategy. ${ }^{12}$ A different way of achieving the properties wanted is by tuning the aggregation state of DPP using other noncovalent interactions, such as H-bonds, in combination with the present $\pi-\pi$ stacking. DPPs are considered H-bonded pigments due to the presence of unsubstituted amides in their structure. Such groups are normally alkylated to provide solubility and hence, unavailable to form H-bonds. However, H-bonding groups can be introduced in different parts of the DPP molecule, like in the periphery of the aromatic rings usually attached to the central core or even as a part of the solubilizing tails pending from the amide groups. In this review, we 
will focus on the work done in hydrogen-bonded DPP derivatives and the influence these noncovalent forces have in the optoelectronic properties, device morphology and effect in the final device outcome. A guide from the discovery of DPP dyes, their properties arising from H-bonding formation and the influence of such noncovalent forces on organic electronic devices will be done.

\subsection{Discovery of diketopyrrolopyrrole}

DPP derivatives were first reported in 1974 as one of the products of a modified Reformatsky reaction that aimed and failed to produce azetinone derivatives (Scheme 1). ${ }^{6}$ Farnum et al. attempted to optimize the reaction conditions to increase the yield of phenyl-functionalized DPP (DPP-Ph, scheme 1), while additionally looking for an elucidation of its formation. Nonetheless, the yield never exceeded $20 \%$, stopping their work on the subject and publishing the results as "the discovery of a pigment chromophore." 6

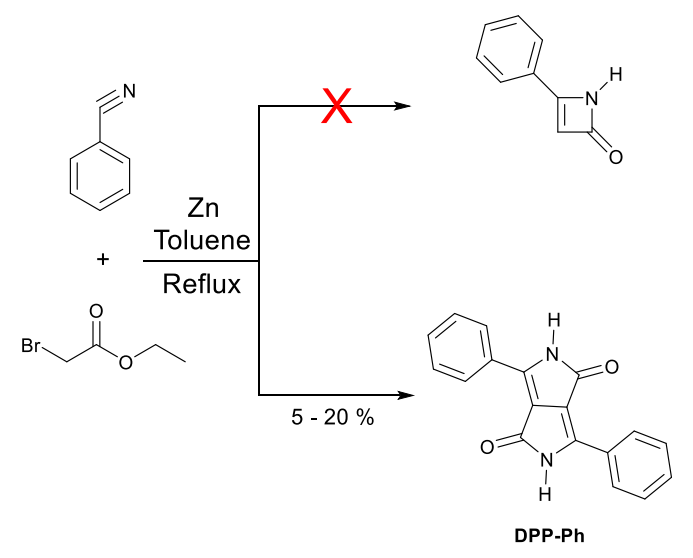

Scheme 1 First and accidental synthesis of DPP-Ph.

The chemical structure of a 2,5-Dihydropyrrolo[4,3-c] pyrrolo-1,4-dione molecule is shown in figure 1 and up to now, this structure has not been reported to be synthesized. The most commonly known DPP derivatives generally have aromatic substituents at the -3 and -6 positions, although compounds with aliphatic groups are also known. ${ }^{13,14}$ Their physical properties such as their high melting point $\left(>350{ }^{\circ} \mathrm{C}\right)$, high insolubility and prominent red color would have been most probably forgotten, if it had not appealed 
the attention of researchers from Ciba-Geigy AG (currently part of BASF), who came across a compilation of interesting reactions published by Ranganathan in $1980 .{ }^{15}$

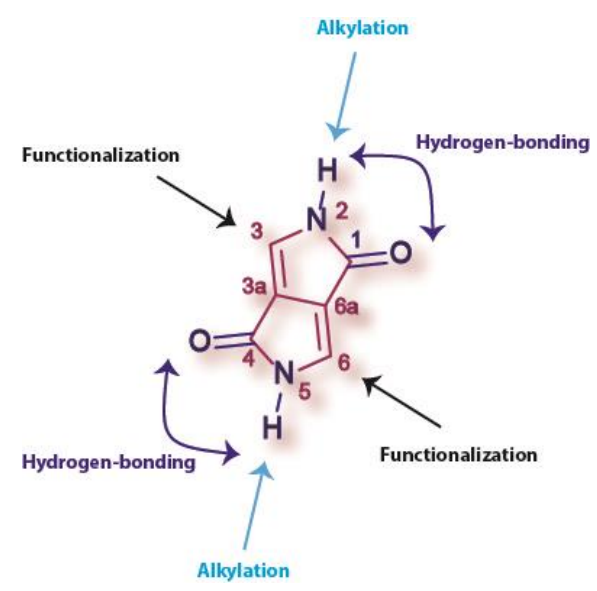

Figure 1. Chemical structure of the DPP core with its corresponding atom numeration, hydrogen-bonding groups and main functionalization positions.

Afterwards, the chemistry along with the applications were developed, and in 1986 the first DPP pigment was introduced to the market. ${ }^{16,17}$ Since then, several other DPP pigments have been presented to the market for conventional pigment applications such as fibers, inks, paints and plastics. ${ }^{18}$ The interest in their high tech applications has grown increasingly in recent years, as DPP derivatives exhibit remarkable resistance against chemical, heat, light and climate stimuli, resulting in more than 9000 patents in different areas. ${ }^{19}$ While used in many fields, the majority of DPP articles comprehend the use of DPP derivatives in semiconductor electronic devices, such as solar cells, ${ }^{7,20}$ organic field effect transistors $(\text { OFETs) })^{9,21}$ and organic light-emitting diodes (OLEDs). ${ }^{22-25}$ Many review articles addressing the applications and synthesis of DPPs have been published, ${ }^{20,22,26}$ although to our knowledge thus far there is not a review that covers the application of H-bonded DPP derivatives. This review will primarily address the works that have been performed with H-bonded DPP structures, as well as their potential application 
for optoelectronic devices and energy applications. Prior to this we considered that the main synthetic approaches of DPP, along with their chemical modifications should be addressed.

\section{Synthesis of diketopyrrolopyrroles}

In this section we will briefly discuss the more common methods used to synthesize DPP derivatives, even though more detailed reviews on this topic can be found in literature. ${ }^{26}$

\subsection{The Reformatsky approach}

A first mechanism was proposed by Farum et al. ${ }^{6}$, which was later proven to be incorrect and revised by researchers from Ciba-Geigy. ${ }^{27}$ The revised mechanism is shown in scheme $2 .{ }^{26}$ As depicted, ethyl bromoacetate reacts with benzonitrile to give salt $\mathbf{1}$, which is later alkylated by a second molecule of ethyl bromoacetate to produce intermediate $\mathbf{2}$, which undergoes cyclization leading to lactam $\mathbf{3}$, subsequently subjected to nucleophilic addition by the cyano group of benzonitrile generating derivative 4 . Lastly, the second lactam ring undergoes closure forming DPP-Ph (5). The best reported yields through this procedure were around $30 \%$ relative to ethyl bromoacetate and $60 \%$ relative to the nitrile. ${ }^{16,17,27}$ Later the reaction conditions were improved using a microwave reactor and reaching yields between $40-70 \%{ }^{28}$ However, this technique has been limited to scarce examples. ${ }^{6,16,17,28}$

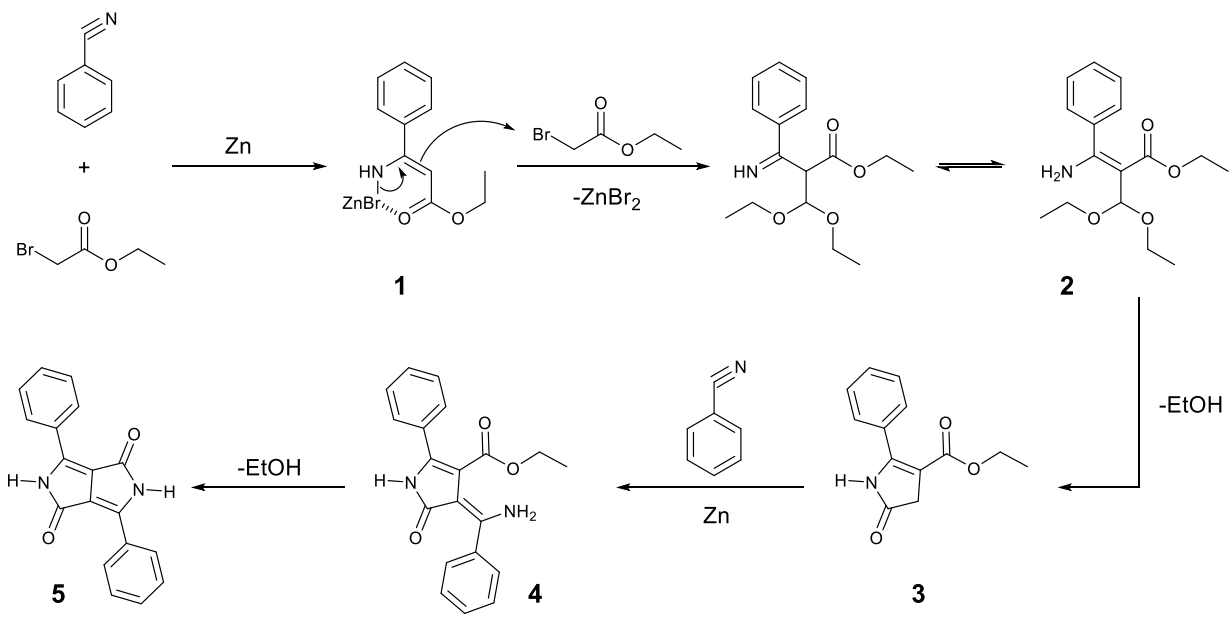

Scheme 2. Proposed mechanism of DPP-Ph formation through the Reformatsky route by Iqbal et al. ${ }^{26}$ 


\subsection{Synthesis by condensation of nitriles through succinic acid esters}

Discovered and developed by investigators at Ciba-Geigy, base-promoted condensation of nitriles with succinic acid esters remains the principal and commonly used technique for the synthesis of DPPs. ${ }^{23,27-30}$ Iqbal et al. discovered that in the presence of alkali metal alkoxides, benzonitrile reacts with dialkyl succinate to form $\mathbf{5}$. This mechanism is similar to the Reformatsky reaction previously described, but in this case the diester $\mathbf{2}$ is produced straight from the succinate and the nitrile. The reaction conditions have been optimized by employing succinates derived from tertiary or secondary alcohols, accomplishing the reaction in the presence of tertiary alkoxides, and by using tertiary alcohols as solvents (Error! eference source not found. 3$)^{16,27}$

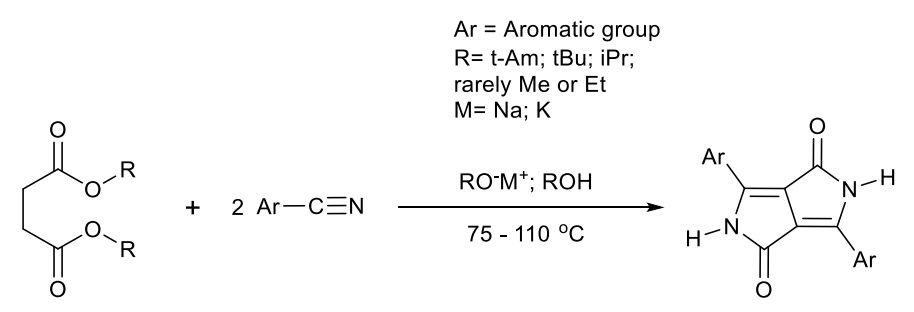

Scheme 3. DPP synthesis by the succinic method.

This synthetic approach holds several advantages, such as the high yields (up to 85\%), simple starting materials, scalability, easy purification, and flexibility to a wide-range of nitriles. ${ }^{27,31}$ Recently, the use of ionic liquids ${ }^{32,33}$ as solvents and the use of microwave irradiation ${ }^{33,34}$ have been reported to aid in the synthesis and be used as alternatives.

\subsection{Synthesis by condensation of nitrile with lactams}

The synthesis of DPP by the succinic method is a good way of achieving a variety of symmetric structures of DPPs. Nonetheless, this approach is inadequate to synthesize asymmetric DPPs carrying two different aryl substituents on positions -3 and -6 (Figure 1). While the condensation of a succinic acid ester along with two different aromatic nitriles can be done, the resulting products are a mixture of three 
DPPs molecules, two that are symmetric and one asymmetric ${ }^{16-18}$ (Error! Reference source not found. ), being difficult to isolate the products due to their poor solubility.

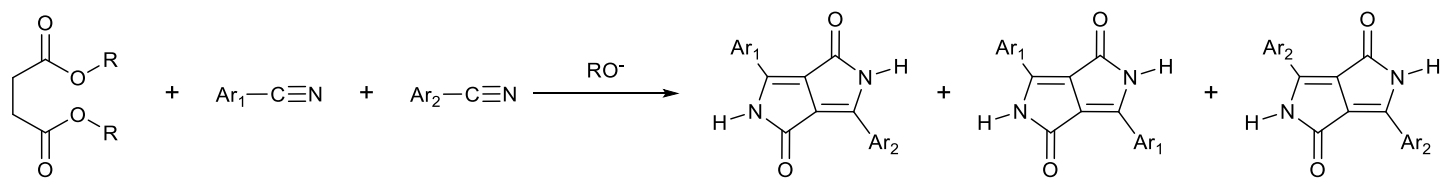

Scheme 4. Mixture of products obtained by the condensation of a succinic acid ester and two different aromatic nitriles.

Even though it is possible to obtain asymmetric DPPs using the Reformatsky approach, the yields are very low and the symmetric product could be also obtained in small amounts. However, the reaction of nitriles with aminoester 2 or lactam 3 (Scheme 2) under basic conditions can lead to asymmetric DPPs. ${ }^{27}$ Scheme 5 shows the reaction of nitriles with dianion 7 (created from a succinic ester) to render aminoester 2 (Scheme 2) and its 2 ' analogues endowed with different $R_{1}$ and $R_{2}$ substituents. In the presence of alkoxides, aminoester 2' and lactam 3' undergo reaction with nitriles leading to the production of DPP (Scheme 5). ${ }^{16,17,27}$

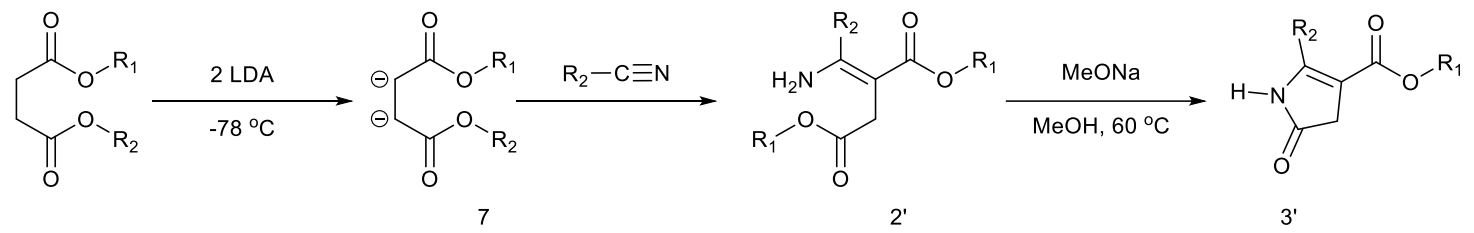

Scheme 5. Synthesis of precursors for the formation of asymmetric DPPs.

This approach is not restricted to diaryl DPP derivatives, DPPs possessing one or two alkyl substituents at positions -3 and -6 can be obtained (Error! Reference source not found. 6). Yet, according to recent iterature, asymmetric DPPs are conceived virtually only from lactams of type 3, ${ }^{35-38}$ 


\subsection{Other synthetic routes}

There are numerous variations to the previously presented methods of DPP synthesis, which can allow for the selective preparation of DPPs holding four different aromatic substituents. ${ }^{39-42}$ Furthermore, there are other synthetic approaches with less relevance, such as the method proposed by Gompper et al, involving the heating of succinic acid diamide with $\mathrm{N}, \mathrm{N}$-dimethylbenzamide diethyl acetal. ${ }^{43,44}$

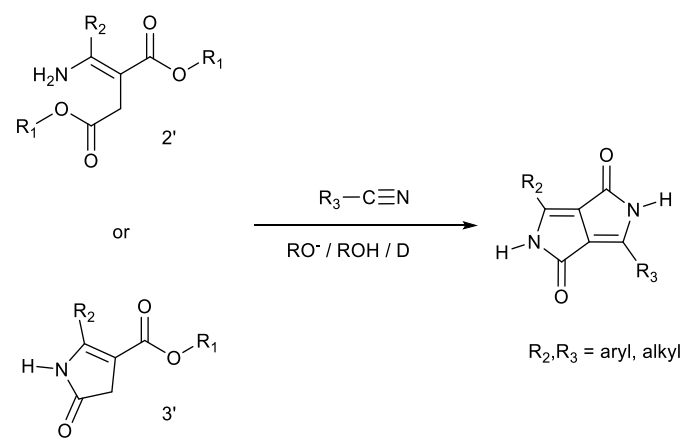

Scheme 6. Synthesis of asymmetric DPPs starting from aminoester 2' or lactam 3'.

\section{Reactivity of diketopyrrolopyrroles}

DPP derivatives contain several reactive groups within their structure susceptible to electrophilic attack, such as oxygen and nitrogen atoms in the amide group, in addition to the double bonds in the DPP core and the aryl groups at the -3 and -6 positions. Conversely, the carbonyl carbon atom or the $\alpha, \beta$ unsaturated system within the bicyclic DPP unit are prone to nucleophilic attack through Michael addition. Furthermore, reactions with nucleophiles can also be performed in the aromatic substituents. In this section, the main chemical reactions that DPP derivatives can undergo will be summarized. For a deeper and extended comprehension, the reader is once again referred to the work of Grzybowski et al. ${ }^{26}$ 


\subsection{Nucleophilic alkylation of the amide groups of DPPS}

While the DPPs with hydrogen atoms at the -2 and -5 positions (Figure 1 and Figure 2) are quite insoluble, alkylation of the nitrogen atom results in very soluble materials. However, DPP derivatives can be alkylated in different positions as shown in figure 2 , depending on the reaction conditions. ${ }^{45}$

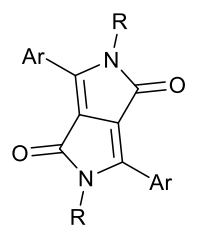

$\mathbf{N}, \mathbf{N}-\mathbf{A l k}$

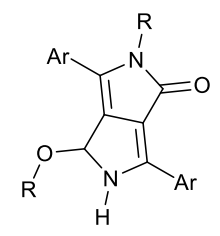

N,O - Alk

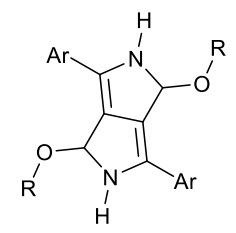

0,0 - Alk

Figure 2. The possible product structures in the alkylation of DPPs.

The O-alkylation is favored in neutral conditions and at low temperature, since the product is kinetically more stable, whereas the $\mathrm{N}$-alkylation is predominantly observed at high temperatures. Thermal rearrangement is also possible when the reaction is performed over long reaction times with excess of alkylation reagents at high temperatures, yielding both alkylated derivatives. ${ }^{46}$ On the other hand, DPPs are deprotonated to give anions under basic conditions, distributing the charges amid the two heteroatoms and promoting the alkylation at either or both $\mathrm{N}$ - and O-atoms. ${ }^{47,48}$ The most common solvents for alkylation are N,N-dimethylformamide (DMF) ${ }^{49,50}$ and N-methylpyrrolidone (NMP), ${ }^{51}$ even though the use of acetonitrile, a greener solvent, has been recently reported, ${ }^{46}$ being possible to run the reaction at room temperature and shorter times.

\subsection{Reactions of the aromatic substituents at the -3 and -6 positions of DPP}

Most DPP derivatives possess aromatic substituents at the -3 and -6 position. Therefore, they may experience the typical reactions of aromatic compounds, especially electrophilic aromatic substitution. ${ }^{16-}$

18 The sulfonation of DPPs is widely used to produce salts used as thermal stabilizer surfactants, improving the rheological properties of DPP paint formulations. ${ }^{16-18,52}$ 
One of the main reactions performed is the halogenation of heteroaromatic substituents in DPPs, such as thiophene, furan, and selenophene. ${ }^{53-55}$ Bromination can be performed by exposing DPPs derivatives to gaseous bromine, but is most frequently done by using N-Bromosuccinimide (NBS), as illustrated on scheme 7.

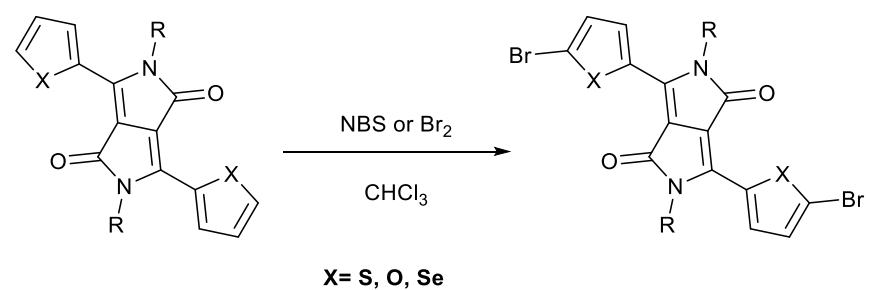

Scheme 7. Bromination of heteroaromatic substituents in DPPs.

DPPs can also be copolymerized to generate biaryl compounds with electron-rich aromatic compounds, due to the electron-accepting character of the DPP unit. Suzuki and Stille couplings are effective procedures for the synthesis of biaryl derivatives ${ }^{56,57}$ The produced copolymers are of the donoracceptor type, having high charge mobility and low-energy bandgap; allowing the material to be applied in semiconducting devices. ${ }^{23,25,58,59}$

Finally, thiophene DPP derivatives can experience reactions with lithium diisopropylAmide (LDA) at the -5 positions of thienyl groups, which can be used to introduce new substituents that can lead to further reactions. ${ }^{60,61}$ A common example of such substitution is shown in scheme 8 , where the formylation leads to dialdehyde DPP derivatives when the reaction is performed with DMF.

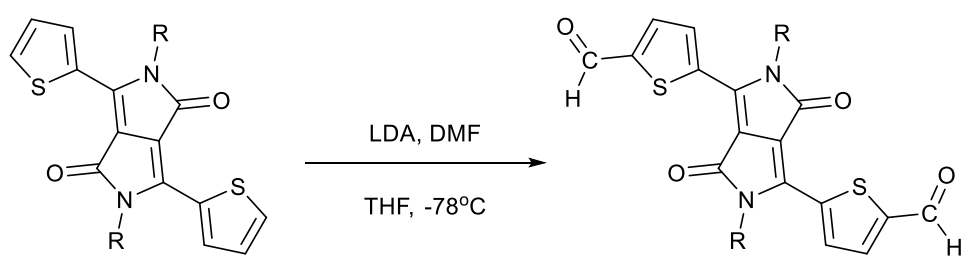

Scheme 8. Formylation of thiophene-DPP derivatives. 
After briefly reviewing the main synthetic routes and reactivity of DPP, the next sections are devoted to the analysis of the effects of hydrogen-bonding found in different parts of the DPP structure. Particularly, their influence in optoelectronic properties, morphology and applications in semiconducting devices, which efficiencies or properties are affected by H-bonding will be discussed.

\section{Hydrogen-bonded DPP with unsubstituted lactam rings}

\subsection{Structural characterization, crystallization and optical properties}

1,4-diketo-3,6-diaryl-pyrrolo -[3,4-c]-pyrrole as synthesized contains two unsubstituted lactams with ability to form hydrogen bonds (Figure 1). In the solid state, there are chains of $\mathrm{NH} \cdots \mathrm{O}$ intermolecular $\mathrm{H}-$ bonds between the $\mathrm{NH}$ group of one molecule and the $\mathrm{O}$ atom of the neighboring molecule forming linear chains. These H-bonds are necessary to align the transition dipole in a "head-to-tail" mode that results in a bathochromic shift of the absorption band when transitioning from the solution state to the solid state. Besides, the NH $\cdots$ O hydrogen bonds connect the DPP molecules to achieve stability similar to those of polymers. The linear chains are parallel to each other and to the plane, while brick wall-pattern $\pi-\pi$ stacking is found perpendicular to the plane (Figure 3). ${ }^{62-64}$ This same structural behavior is seen in other H-bonded pigments, such as indigo, ${ }^{65,66}$ perylene bisimides, ${ }^{67}$ quinacridone ${ }^{68,69}$ or epindolidione, ${ }^{68}$ yet the H-bonding distances were found to be shorter in DPP. ${ }^{70}$ Even though many authors have claimed that amine and carbonyl groups should be avoided in the molecular structures of semiconductors due to a possible disruption of the conjugation, ${ }^{71}$ it has been demonstrated that maximizing the charge transfer integrals through neighboring molecules can enhance the mobility. ${ }^{70}$ In this sense, crystal engineering through hydrogen bonding has been one of the main strategies applied in device fabrication.

Early investigations by Mizuguchi ${ }^{72-79}$ at the end of the 80 's and early 90 's show the crystal and electronic structures of several small H-bonded DPP molecules, starting from 1,4-diketo-3,6-diphenylpyrrolo-[4,4-c]-pyrrole ${ }^{72-74}$ and extending it to the dithioketo, ${ }^{80}$ pyridine-containing $^{81,82}$ and halogenated analogues. ${ }^{75}$ The conclusions of these studies showed that the intermolecular $\mathrm{NH}^{\cdots} \mathrm{O}$ H-bonds result in a bathochromic shift in the absorption maxima upon crystallization with respect to solutions (Figure 4), and 
that the spectral shape in solid state differs substantially from the one in solution mainly depending on the extent of the molecular overlap along the stacking axis. When the molecular overlap is insignificant, the solid state and solution absorption spectra are quite similar and when the molecular overlap increases along the stacking axis, the absorption maximum shows a hypsochromic shift, making the color more yellow.
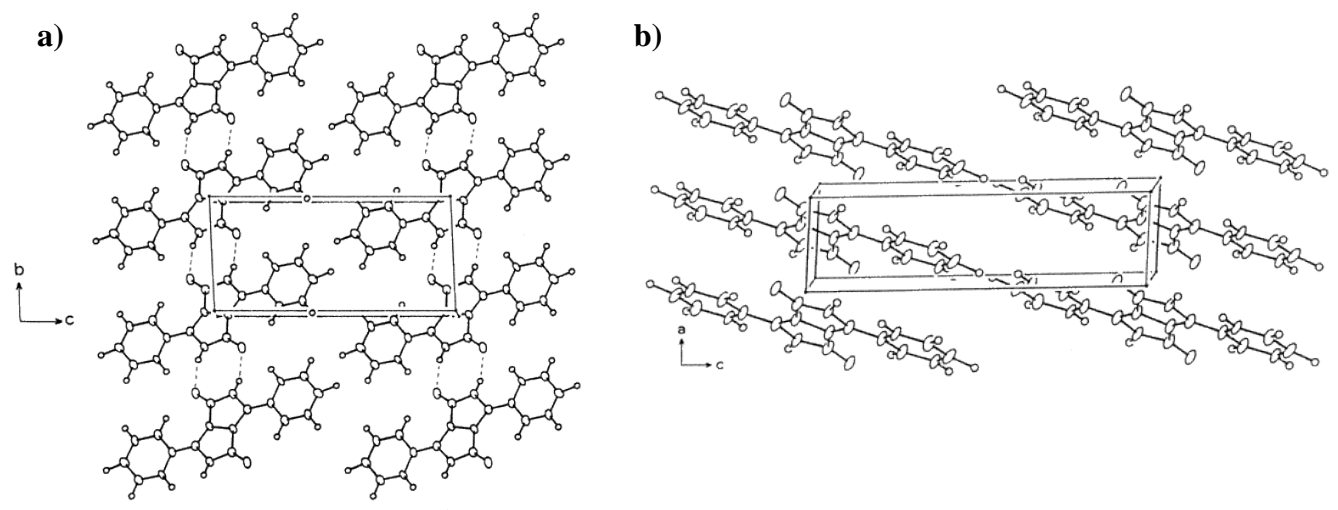

Figure 3. Projection of the crystal structure of DPP a) onto the plane $(b, c), \mathrm{b})$ onto the $(a, c)$ plane.

Reprinted with permission from (J. Mizuguchi, J Phys Chem A, 2000, 104, 1817-1821). Copyright (2000)

American Chemical Society.

The authors concluded that the "head-to-tail" arrangement caused by H-bonding is found to displace the absorption maximum towards longer wavelengths, while the stack pair or parallel arrangement significantly contributes to the hypsochromic shift. ${ }^{62}$ In this work, the exciton coupling model based on the interaction between transition dipoles was used to qualitatively interpret the correlation between the crystal and electronic structures in DPP derivatives. This way, it could be understood why the famous "Ferrari red" pigment ( $\boldsymbol{p}$-CIDPP) shows bright red color mainly due to the formation of the "head-to-tail" arrangement thanks to H-bonding $v s$ the yellowish color in $m$-substituted derivatives, where the stack pair contribution (hypsochromic shift) overpowers the H-bonding. These initial findings led to the study of 
different applications, such as $\mathrm{H}_{2}$ gas sensors, ${ }^{82}$ information storage $^{76}$ and the study of photoconductivity. ${ }^{78}$

Still until very recently the supramolecular ordering of H-bonded DPP pigments have continued to be studied. Perepichka et $a l^{83}$ used scanning tunneling microscopy (STM) combined with X-ray crystallographic analysis to demonstrate how the interactions of heteroatoms in the aromatic substituents of the DPP core interplay with hydrogen-bonding and the final influence on charge transport properties. The authors studied the supramolecular order of H-bonded difuran, dithiophene and diphenyl DPP derivatives in monolayers at the solid-liquid interface and in bulk crystalline solids. This study demonstrated that even though H-bonding is the main and strongest interaction in the supramolecular assemblies of the DPP derivatives described, their structures change radically depending on the nature of the aromatic substituents (furan, phenyl or thiophene). The diphenyl derivative was found to form exclusively H-bonded homoassemblies; the difuran DPP preferentially co-assembles with alkanoic acids (used to create the solid-liquid interface), and the dithiophene substituted DPP either co-assembles with alkanoic acids or self-assembles in one of two $\mathrm{H}$-bonded polymorphs depending on the conditions. Remarkably, one of these two polymorphs shows an out-of-place (in graphite) twist of the thiophene rings that provides for stronger intermolecular interactions and higher molecular density. This was the first case reported of a planar molecule reorganizing into a less favorable twisted geometry and it could help for example in OFET applications, where the interfacial layer plays a crucial role in the device properties. 


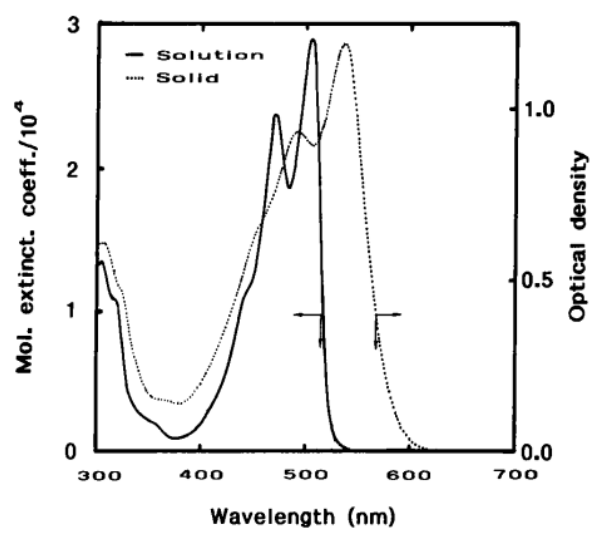

Figure 4: Absorption spectra of DPP-Ph in dimethyl sulfoxide (DMSO) solution and on evaporated thin film. Reprinted with permission from (J. Mizuguchi, J Phys Chem A, 2000, 104, 1817-1821). Copyright (2000) American Chemical Society.

\subsection{Vacuum deposited thin films and the use of the latent pigment strategy}

In 1997, Iqbal et $\mathrm{al}^{84}$ showed a method to achieve soluble DPP derivatives by introducing $t$-Boc (tertbutoxycarbonyl) protecting groups, synthesizing the so-called "latent pigments" that render the final dye after thermal cleavage of the $t$-Boc groups, which decomposes into isobutene and $\mathrm{CO}_{2}$ at approximately $180{ }^{\circ} \mathrm{C}($ Figure 5).

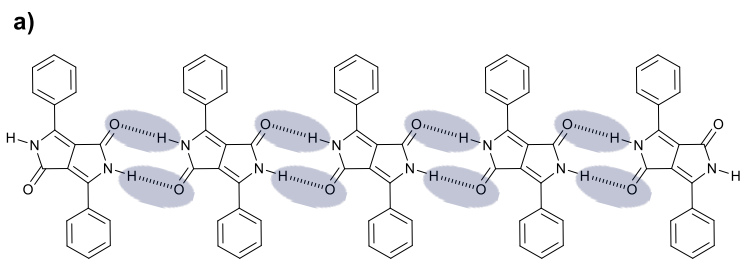

b)

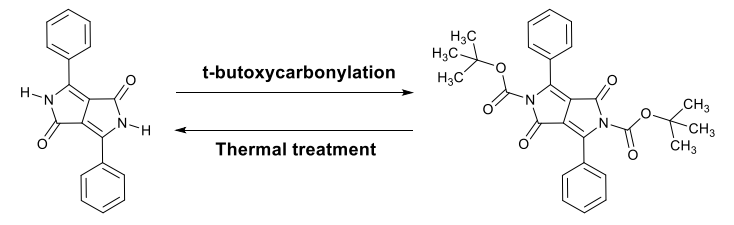

Figure 5: a) Schematic illustration of the H-bonding network of DPP. b) Insoluble parent DPP pigment and soluble latent pigment. Adapted with permission from "J. S. Zambounis, Z. Hao and A. Iqbal, Nature, 1997, 388, 131”. Copyrights 1997, Springer Nature. 
Later on, this technique has been widely used for the fabrication of thin films cast from solution, which is an easier and cheaper method than vacuum deposition techniques, even though several parameters such as removal rate, the nucleation and growth mechanism of the thermally converted derivatives and the role of the volatilized molecules needed to be explored. Several studies have been reported on the influence of the solubilizing group removal on the morphology and properties of H-bonded DPPs. For instance, Salammal $e{ }^{8} l^{85}$ studied the role of the solubilizing group removal rate on the grain size and crystallinity of DPP-4T (Figure 6). The authors studied the use of different heating rates ranging from 0.1 to 50 ${ }^{\circ} \mathrm{C} /$ min until reaching $225^{\circ} \mathrm{C}$, and the isothermal decarboxylation of the DPP-4T precursor at different temperatures. In this case, it was found that the crystallite size increased from 344 to $976 \mathrm{~nm}$ when the precursor film was heated up to $225{ }^{\circ} \mathrm{C}$ increasing the heating rate from 0.1 to $50{ }^{\circ} \mathrm{C} / \mathrm{min}$, while improving the crystallinity as well. This result could be explained due the formation of H-bonding between decarboxylated molecules during the removal process. If the heating rate is too low, the $t$-Boc groups not cleaved can hinder the formation of $\mathrm{H}$-bonds $(\mathrm{N}-\mathrm{H} \cdots \mathrm{O})$ with neighboring molecules, decreasing the crystallite size and crystallinity due to the disruption in growth in one dimension and trapping of $t$-Boc in the network. On the other hand, when the heating rate or the isothermal deprotection temperature increase, the volatilized isobutene and $\mathrm{CO}_{2}$ can scape easily from the network, and the growth of crystallites is facilitated by the simultaneous decarboxylation of both $t$-Boc groups that results in the formation of H-bonds. 


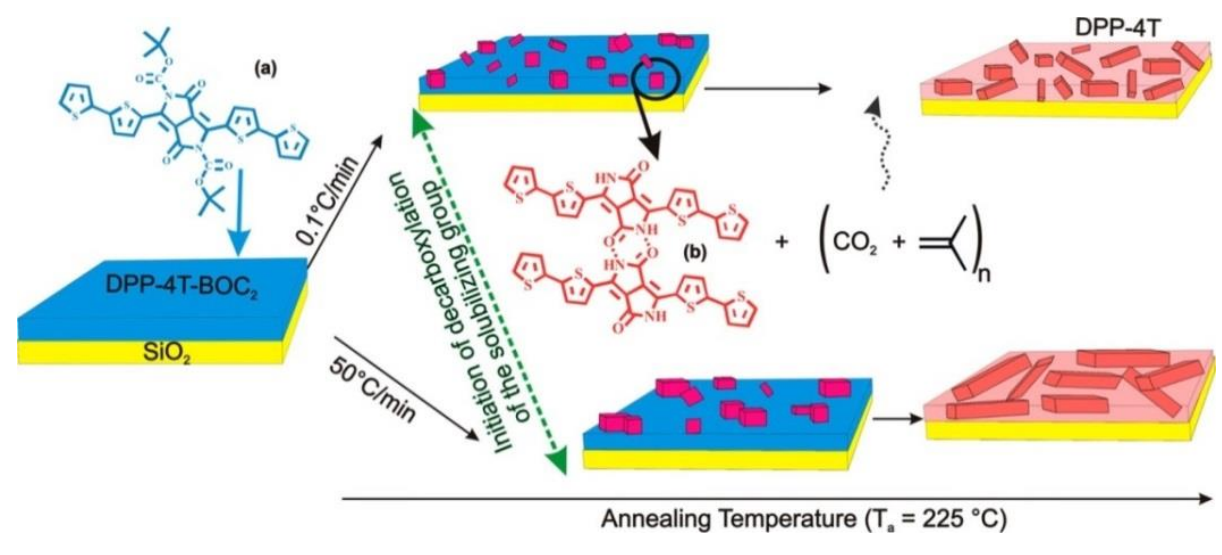

Figure 6: Schematic representation of the impact of the heating rate on morphology and final grain size. Reprinted with permission from (S. T. Salammal, J.-Y. Balandier, S. Kumar, E. Goormaghtigh and Y. H. Geerts, Cryst. Growth Des., 2014, 14, 339-349). Copyright (2014) American Chemical Society.

One of the first examples of electronic devices using thermal removal of solubilizing groups was reported by Sakai et al in 2008. ${ }^{69}$ This work reports the use of phenyl substituted DPP using the $t$-Boc-protected latent pigment that is soluble in common organic solvents. OFETs were fabricated by spin coating the latent pigment and by thermally annealing at $180{ }^{\circ} \mathrm{C}-200{ }^{\circ} \mathrm{C}$ for 15 minutes, to yield the deprotected DPP able to form H-bonds. Mobility values in the order of $10^{-6} \mathrm{~cm}^{2} \mathrm{~V}^{-1} \mathrm{~s}^{-1}$ were obtained, which were similar $\left(10^{-5} \mathrm{~cm}^{2} \mathrm{~V}^{-1} \mathrm{~s}^{-1}\right)$ to the ones obtained by vacuum depositing the same type of material. The authors reported a difference in morphology when films were spin coated from solution and then the H-bonds were thermally regenerated, and when the H-bonded pigments were vacuum deposited. In the first case, the films studied by scanning electron microscopy (SEM) showed a rough and uneven surface with needle-like structures $v s$ a very smooth surface when the DPP derivatives were vacuum deposited.

The authors demonstrated the simplicity of the preparation method but later, Yamashita et $l^{86}$ reported that the mobility values in the work of Sakai could be due to the production of $\mathrm{CO}_{2}$ and isobutene released during the thermal annealing process, which negatively impacted the morphology of the spin 
coated film. The work of Yamashita shows larger DPP derivatives (Figure 7) for the fabrication of OFETs using the same strategy. In this case, OFETs that showed ambipolar charge-carrier transfer with field effect mobility values of $\mu_{\mathrm{h}}$ of $6.7 \times 10^{-3} \mathrm{~cm}^{2} \mathrm{~V}^{-1} \mathrm{~s}^{-1}$ and $\mu_{\mathrm{e}}$ of $5.6 \times 10^{-3} \mathrm{~cm}^{2} \mathrm{~V}^{-1} \mathrm{~s}^{-1}$ were fabricated. The optical properties of NH BTTDPP1 and NH BTTDPP2 change dramatically when comparing solution to thin films (Figure $7 \mathrm{~b}$ and $7 \mathrm{c}$ ), initially just by spin coating and subsequently, by thermally annealing and cleaving the $t$-Boc groups. A bathochromic shift of $80 \mathrm{~nm}$ is observed in the absorption onset of NH BTTDPP1, while in NH BTTDPP2 the absorption onset red-shifts more than $200 \mathrm{~nm}$. In both cases, a shoulder band at lower energy appears, that dramatically increases after thermal treatment and that is related to the formation of H-bonds. The authors use infrared (IR) measurements to confirm the appearance of H-bonds on the thermally treated films, being this technique fundamental in these types of studies. OFET devices were fabricated and field-effect mobility values were measured in devices made with the latent pigments and with H-bonded films. In the first case, hole mobility values in the order of $10^{-6}$ and $10^{-5} \mathrm{~cm}^{2} \mathrm{~V}^{-1} \mathrm{~s}^{-1}$ were found (similar to the studies of Sakai years before) and that were enhanced by two orders of magnitude after thermal cleavage of the $t$-Boc groups. Interestingly, derivative NH BTTDPP2 containing two DPP moieties in the structure, exhibited well-balanced hole and electron mobilities in the order of $10^{-3} \mathrm{~cm}^{2} \mathrm{~V}^{-1} \mathrm{~s}^{-1}$. Previously, a H-bonded DPP system containing electron withdrawing $-\mathrm{CF}_{3}$ groups in para-position used as n-channel semiconductors with electron mobility values of $2.9 \times 10^{-2} \mathrm{~cm}^{2} \mathrm{~V}^{-1} \mathrm{~s}^{-1}$ were reported by this group. ${ }^{87}$ In this case, the devices were fabricated by vacuum deposition directly from the H-bonded pigment, while the latter study was the first one finding ambipolar behavior using hydrogen-bonded DPP molecules and processing the devices from solution. 
a)

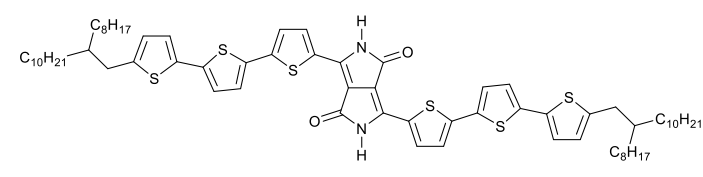

NH BTTDPP1

b)

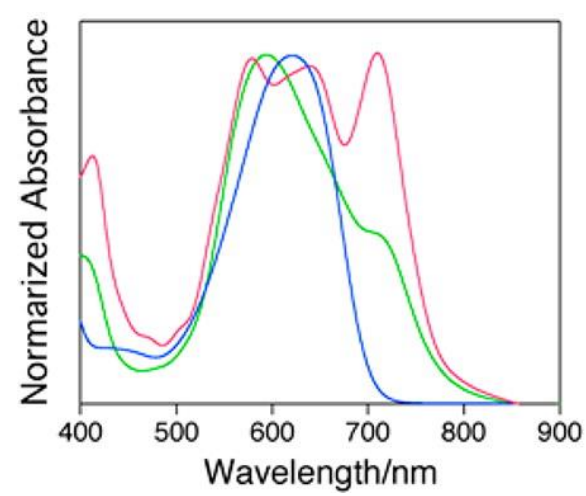

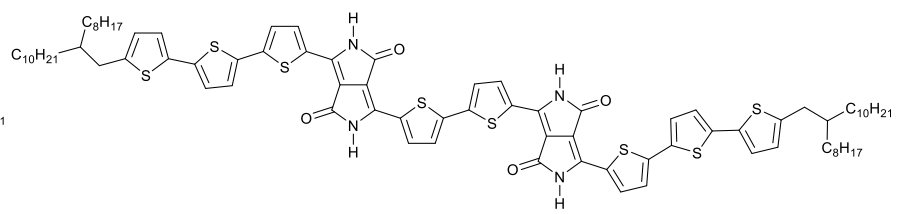

NH BTTDPP2

c)

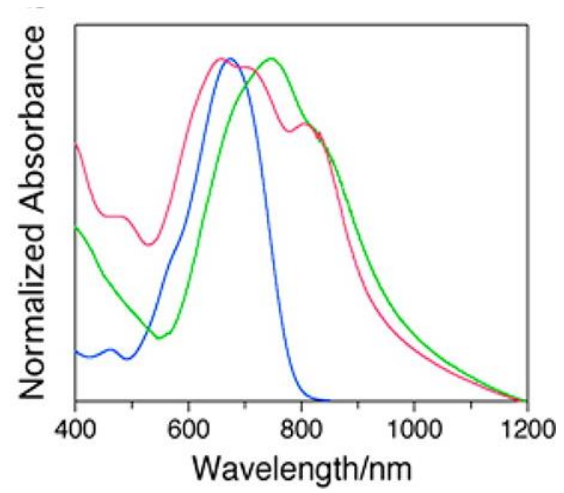

Figure 7: a) Molecular structures of NH BTTDPP1 and NH BTTDPP2. b) UV-Vis spectra of Bocproteted NH BTTDPP1 in solution (green trace), thin film (green trace), and NH BTTDPP1 (red trace). c) UV-Vis spectra of Boc-protected NH BTTDPP2 in solution (green trace), thin film (green trace), and

NH BTTDPP2 (red trace). Reprinted with permission from (Y. Suna, J. Nishida, Y. Fujisaki and Y. Yamashita, Org. Lett., 2012, 14, 3356-3359). Copyright

(2012). Jon Wiley and Sons.

Other reports using vacuum deposition techniques of H-bonded DPPs present archetypical derivatives (Figure 8a) containing in this case halogen (-Cl and -Br) substituents in the -para position of the phenyl rings that decorate the DPP core. ${ }^{70}$ In this work, the results are complemented with density functional theoretical (DFT) calculations to better understand the charge transport studies. The authors used anodically-grown $\mathrm{AlO}_{x}$ passivated with tetratetracontane $\left(\mathrm{C}_{44} \mathrm{H}_{90}\right)$ as a composite low surface-energy dielectric to fabricate OFET devices because they found out in previous works on indigo dyes that low 
surface-energy dielectrics were crucial to enhance high mobility in H-bonded small molecule devices. ${ }^{88}$ The final mobility values were enhanced several orders of magnitude with respect to the initial report by Sakai et al, ${ }^{69}$ who used phenyl-substituted DPP as well. Furthermore, ambipolar behavior was reported for the three hydrogen-bonded DPP derivatives shown, being $\mu_{\mathrm{h}}=\mu_{\mathrm{e}}=0.01 \mathrm{~cm}^{2} \mathrm{~V}^{-1} \mathrm{~s}^{-1}$ for DPP-Ph, $\mu_{\mathrm{h}}$ of 0.03 and $\mu_{\mathrm{e}}$ of 0.01 for the $\boldsymbol{p}$-Cl DPP and $\mu_{\mathrm{h}}$ of 0.06 and $\mu_{\mathrm{e}}$ of 0.02 for $\boldsymbol{p}$-Br DPP. The crystalline structures obtained are very important to understand the differences in mobility values. While in the phenyl derivative, the H-bonded chains are parallel to one another (Figure 8b) and parallel to the plane, which was the same for the para-halogenated DPPs but the chains were staggered relative to each other making two linear H-bonded chains run along the [001] plane, and other two chains run along the [002] plane, with a tilt with respect to the first chain (Figure 8c). The crystal structures were described as pseudo brick-wall $\pi$ - $\pi$ stacking. While no discrepancies in charge transport were expected for the three derivatives described, a difference in crystallite size was found. DPP-Ph formed crystal grains between 100-200 nm with clearly defined boundaries, $\boldsymbol{p}$-CI DPP had similar crystallites but oblong in shape, and $\boldsymbol{p}$-Br DPP had smaller grains making very smooth films and more continuous. The higher mobility values found for $\boldsymbol{p}$-Br DPP might be explained according to this morphology.

a)

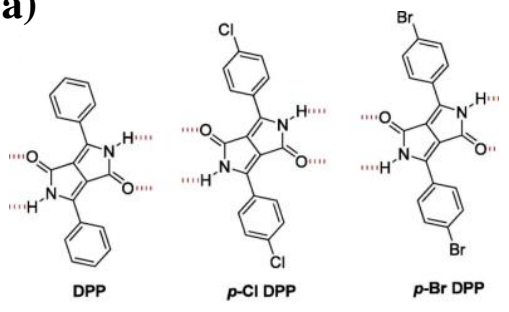

b)

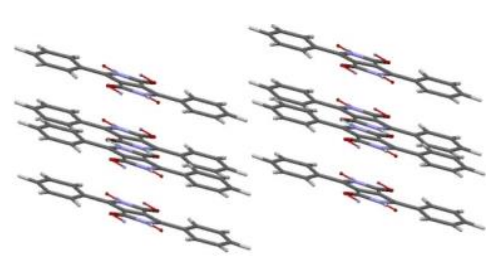

c)

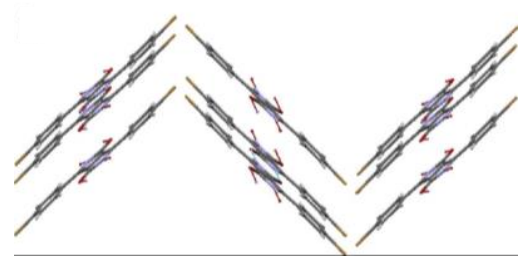

Figure 8. Molecular structures of a) DPP, b) $p$-Cl DPP and c) $p$-Br DPP. ${ }^{70}$

To demonstrate the strength of this strategy, more sophisticated oligomers have been reported recently, as for example the work of Zhu et al., ${ }^{89}$ where two DPP oligomers with different number of phenyl and thiophene rings were attached to the central DPP core, which was functionalized with thermo labile 
groups were based on 2-methylhexyl-2-oxylcarbonyl. In this case, field effect behavior was only found for the derivative containing thiophene rings (HTBT), including its latent pigment. Other examples as the work of Mula et al. ${ }^{90}$ showing thiophene-capped DPP coupled to triazatruxene (TAT) derivatives (Figure 9) with $\mu_{\mathrm{h}}$ of $4.2 \times 10^{-4} \mathrm{~cm}^{2} \mathrm{~V}^{-1} \mathrm{~s}^{-1}$. Interestingly, the charge transport properties were unaffected when blends of NH-TATDPP and phenyl [6,6]-Phenyl $\mathrm{C}_{71}$ butyric acid methyl ester $\left(\mathrm{PC}_{71} \mathrm{BM}\right)$ were studied, emphasizing the robustness of the morphology when NH-TATDPP was used as donor material.

The use of thermocleavable side chains has been applied in semiconductors containing longer solubilizing chains, especially when using polymers. Sun et $a l^{91}$ reported a DPP-based polymer containing 2-octyldodecanoyl side chains that can be thermally removed at $200{ }^{\circ} \mathrm{C}$ to generate a side chain-free conjugated polymer. X-ray diffraction (XRD) measurements and atomic force microscopy (AFM) images show the thin film progress upon thermal decomposition of the side chains. The as-spun films showed a diffraction peak at $2 \Theta$ of $4.40^{\circ}$, which corresponds to a $d$-spacing of $20.1 \AA$ and that was attributed to the inter-lamellar distance. Upon raising the temperature, the primary peak of the thin film became more intense as a result of the improved molecular ordering by thermal annealing. When reaching $200{ }^{\circ} \mathrm{C}$ for 3 hours, the main diffraction peak disappeared as a result of the total elimination of the side chains, which resulted in the shortening of the interlayer distance. AFM images showed that at $150{ }^{\circ} \mathrm{C}$ the films were quite smooth, while at $200{ }^{\circ} \mathrm{C}$ for 3 hours, the nanograins initially formed evolved into connected nanofibers with higher roughness and large gaps between domains. When the charge transport properties were studied, the hole mobility values when annealing at $150{ }^{\circ} \mathrm{C}\left(\mu_{\mathrm{h}}=0.096 \mathrm{~cm}^{2} \mathrm{~V}^{-1} \mathrm{~s}^{-1}\right)$ were one order of magnitude lower than the mobility of the polymer with solubilizing alkyl chains previously reported. ${ }^{92}$ Still at $200{ }^{\circ} \mathrm{C}$, the mobility values $\left(\mu_{\mathrm{h}}=0.078 \mathrm{~cm}^{2} \mathrm{~V}^{-1} \mathrm{~s}^{-1}\right)$ were lower than at $150{ }^{\circ} \mathrm{C}$. The authors concluded that the amorphous nature of the decarboxylated polymer needs to be taken into account, being in this case an efficient material for charge transport. 

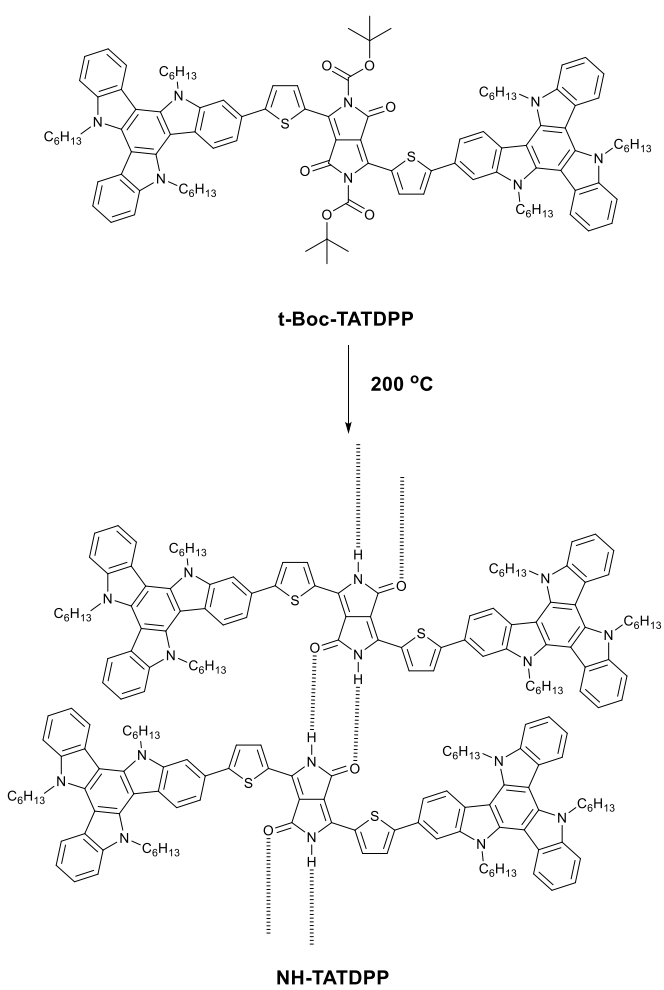

Figure 9. Synthesis of compound NH-TATDPP. Reprinted with permission from (S. Mula, T. Han, T. Heiser, P. Lévêque, N. Leclerc, A. P. Srivastava, A. Ruiz-Carretero and G. Ulrich, Chem. - Eur. J., , DOI:10.1002/chem.201900689). Copyright (2019). Jon Wiley and Sons.

Other DPP polymers with thermocleavable groups can be found in literature. Lee et $a l^{93}$ for example, reported inversion of the dominant polarity in H-bonded DPP derivatives upon thermal treatment using low bandgap polymers (Boc-PTDPP, figure 10) in this case. In this work, OFET devices were fabricated applying a solution-sheared deposition technique, where a volume of the H-bonded DPP was placed between two preheated silicon wafers that move relative to each other at a specific rate (Figure 10b). This way, the semiconductor molecules can form highly crystalline and elongated grains along the shearing direction. The authors compared drop cast and solution-sheared films using IR spectroscopy before and after thermal annealing. The $\mathrm{C}=\mathrm{O}$ stretching signal disappears after thermal treatment with a concomitant 
appearance of the N-H band as a consequence of the carbamate deprotection. As an indication of the formation of $\mathrm{H}$-bonds, the $v_{\mathrm{C}=\mathrm{O}}$ (amide) shifted to lower energies. Remarkably, the solution-sheared films showed further shift of the $v_{\mathrm{C}=\mathrm{O}}$ (amide) compared to the drop cast films, probably due to stronger $\mathrm{H}$ bonding in the solid state in these types of films. The ambipolar Boc-PTDPP was integrated into transistors and showed p-channel dominant characteristics, resulting in $\mu_{\mathrm{h}}$ and $\mu_{\mathrm{e}}$ of $1.32 \times 10^{-2} \mathrm{~cm}^{2} \mathrm{~V}^{-1} \mathrm{~s}^{-1}$ and $2.3 \times 10^{-3} \mathrm{~cm}^{2} \mathrm{~V}^{-1} \mathrm{~s}^{-1}$, respectively. These values are one order of magnitude higher than the values found in devices made by drop-casting the polymer. After decarboxylation at $200{ }^{\circ} \mathrm{C}$ the dominant polarity of charge carriers changed from positive to negative in devices fabricated by solution-shearing, reaching $\mu_{\mathrm{e}}$ of $4.6 \times 10^{-2} \mathrm{~cm}^{2} \mathrm{~V}^{-1} \mathrm{~s}^{-1}$ and decreasing $\mu_{\mathrm{h}}$ in one order of magnitude. DFT studies suggest that the LUMO orbitals of the deprotected polymer become much more delocalized than the protected polymer, which is favorable for $n$-channel conduction. Furthermore, the downshift of the HOMO-LUMO levels after thermal treatment could decrease the injection barrier for holes. The authors also suggest that the removal of $t$-Boc groups could act as electron traps and facilitate electron transport. 


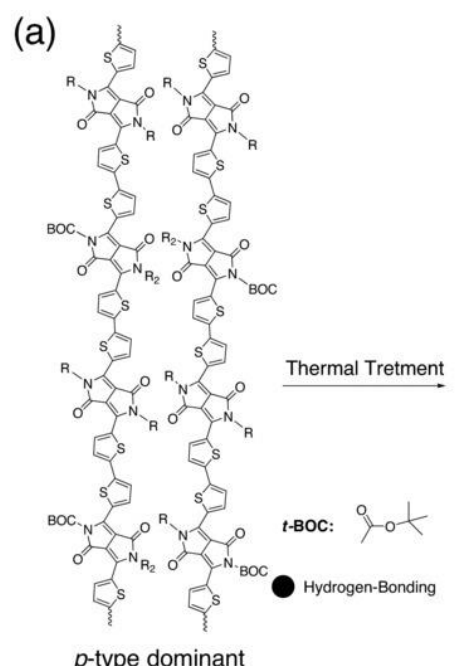

p-type dominant

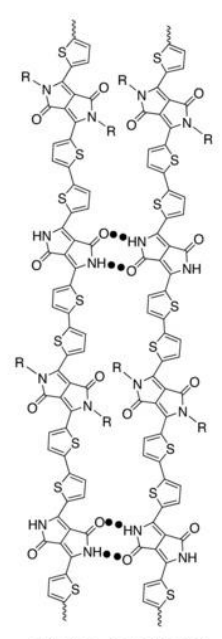

n-type dominant (b) Shearing direction

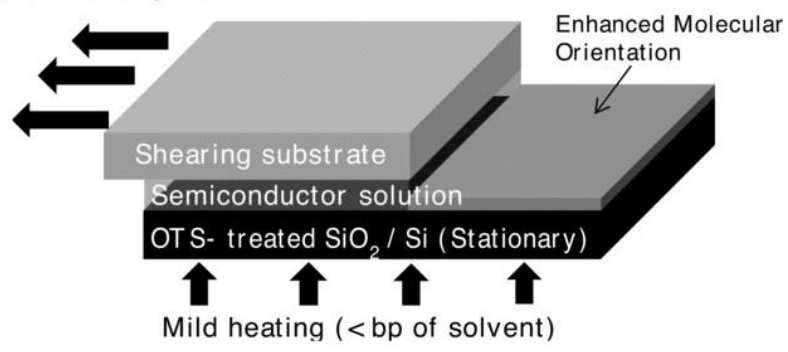

Figure 10. a) Thermocleavable polymer based on DPP. (b) Schematic illustration of solution-shearing technique. Reprinted with permission from "J. Lee, A.-R. Han, J. Hong, J. H. Seo, J. H. Oh and C. Yang, Adv. Funct. Mater., 2012, 22, 4128-4138”. Copyright 2012. Jon Wiley and Sons.

Even though most of the examples report the fabrication of OFET devices, some reports can be found on the fabrication of bulk heterojunction solar cells (BHJSCs). Brovelli et al. ${ }^{94}$ used this strategy to optimize the charge separation efficiency at the donor/acceptor interface in an attempt to form interpenetrated phase separated percolation pathways. Proof-of-principle devices consisting of $100 \mathrm{~nm}$ blends of $t$-Boc protected DPP-Ph and PCBM were fabricated and the performance was followed at different thermal treatment times. While the open circuit voltage $\left(V_{O C}\right)$ showed no dependence on increasing the thermal treatment time from 0 to $300 \mathrm{~s}$, the short circuit current $\left(J_{S C}\right)$ showed a 30 -fold increase (reaching its maximum at $270 \mathrm{~s}$ of treatment time), resulting in a 20 -fold enhancement of efficiency with respect to the pristine device. The morphology of the devices was followed by AFM and XRD during the thermal treatment and it was observed that the films changed from being uniform with roughness below $1 \mathrm{~nm}$ at the initial state, to an increase in crystallinity and formation of 10-20 nm threedimensional crystalline domains at $270 \mathrm{~s}$ of thermal treatment. The blends were stored in air under 
ambient illumination for up to 6 months, finding the same morphological features and emphasizing the strength of this strategy to stabilize the nanoarchitecture of the blend. Control experiments were carried out using a DPP containing alkyl tails (ethylhexyl) and interestingly, the efficiency values were lower emphasizing the effect of the hydrogen-bonding strategy.

\subsection{Mono-alkylated DPPs}

Even though the DPP with unsubstituted amides lack solubility and should be processed via vacuum deposition or by the latent pigment technology, the mono-alkylated DPP derivatives show very interesting properties while combining solubility and ability to form $\mathrm{H}$-bonds. Patil et al, ${ }^{95}$ reported mono-hexyl phenyl and mono-hexyl thiophene DPP derivatives and studied their properties by single crystal X-ray analysis and correlated the results to the charge transport properties in both, mono and dialkylated analogues. The authors found that while the di-alkylated analogues show herringbone packing arrangements, the mono-alkylated derivatives crystallize in a co-facial layered structure thanks to the formation of intermolecular H-bonding between the free amide groups (Figure 11a). Charge carrier mobility values (p-type) two orders of magnitude higher were found for the mono-alkylated derivatives compared to the di-alkylated analogues. The authors explain that according to the crystal structures and theoretical calculations, charge transport occurs in two or three directions while it happens in only one direction for the di-alkylated derivatives.

Pop and Amabilino showed similar results extending their study to a larger variety of di- and mono-

alkylated thiophene-capped DPPs. ${ }^{96}$ In this case, the authors found out herringbone structures in some of the mono-alkylated DPP derivatives. Particularly, the authors found out that the mono-substituted DPPs with hexyl and ethyl acetate substituents were less distorted than the other derivatives of the series, causing stronger intermolecular H-bonding that increases the molecular overlap and planarity of the DPP cores. More specifically, the mono-substituted DPP with ethyl acetate showed high potential for optimizing the charge transport properties in be applied in organic electronic devices. 
a)

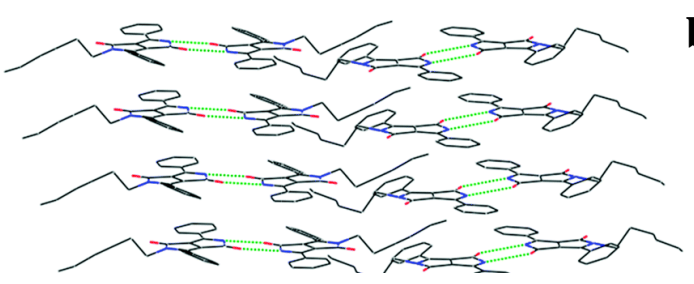

PDPP-MH

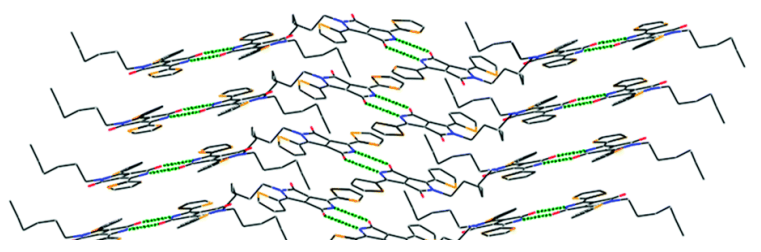

TDPP-MH b)
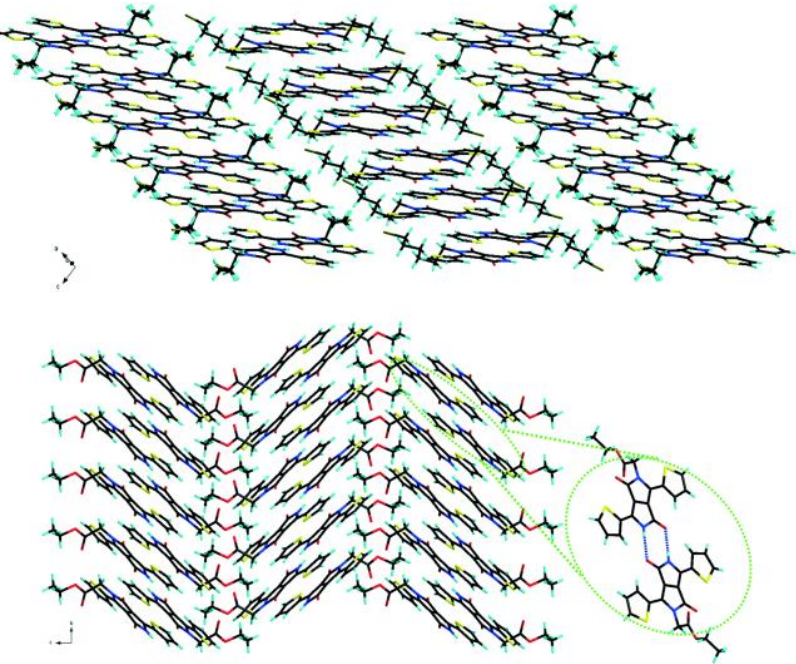

Figure 11. a) Co-facial packing via H-bonding in PDPP-MH and TDPP-MH. b) Molecular packing of two of the studied mono-alkylated DPP derivatives, one showing anisotropy of the p-stacking along the $a$ axis (top) and another one showing co-facial herringbone arrangement (bottom).

Other examples of mono-alkylated DPP derivatives have been reported in literature but most of them have been applied as anion sensors thanks to the binding of anions by $\mathrm{H}$-bonding with the free amide in one of the lactam rings. ${ }^{97-99}$

\section{DPP systems with H-bonding groups in different positions: polymers and small molecules}

Even though the DPP pigment is considered an H-bonded pigment as shown in the previous section, it can only be processed by vacuum deposition or using the latent pigment technology after applying thermal or acid treatment. Due to the possibility of functionalization of the DPP core, it is possible to introduce different types of H-bonding motifs in soluble derivatives, making possible to study the aggregation in solution as well as device fabrication. 


\subsection{Amide-functionalized DPP systems}

One common strategy for introducing H-bonds into the molecular structure of DPP derivatives is attaching amide groups at the periphery of the aromatic rings attached to the central DPP core. This strategy is usually based on introducing an aldehyde group that can react subsequently with electrondeficient acetamides via Knoevenagel condensation. In this sense, most of the examples found in literature are based on linear molecules, even though some cases of hairpin-shaped DPP derivatives are

also reported. An example of the latter approach was reported by Stupp et al, ${ }^{100}$ where a hydrogen-bonded small molecule containing trans-1,2-diamidocyclohexane and two DPP conjugated arms (Figure 12). s were fabricated with the hairpin derivative DPPHP as the donor material, and $\mathbf{P C} \mathbf{C}_{\mathbf{7 1}} \mathbf{B M}$ as the acceptor, finding that a stepwise self-assembly process was needed to achieve functional devices (Figure 12). When both components were mixed in solution in an attempt to simultaneously assemble them into organized structures, non-working devices were found (Figure 12, top). On the other hand, a slow cooling process starting from the molecularly dissolved state of the hairpin-shaped derivative (Figure 12, bottom), yielded robust supramolecular wires that were not disrupted when $\mathbf{P C}_{71} \mathbf{B M}$ was added, resulting in well-defined heterostructures with improved efficiency. The self-assembly processes were followed by cryogenic transmission electron microscopy (cryo-TEM) and AFM, observing the presence of nanofibers in solution that were still present after the addition of $\mathbf{P C}_{71} \mathbf{B M}$ and visible on the solar cell active layer (Figure 12A, B and C). 


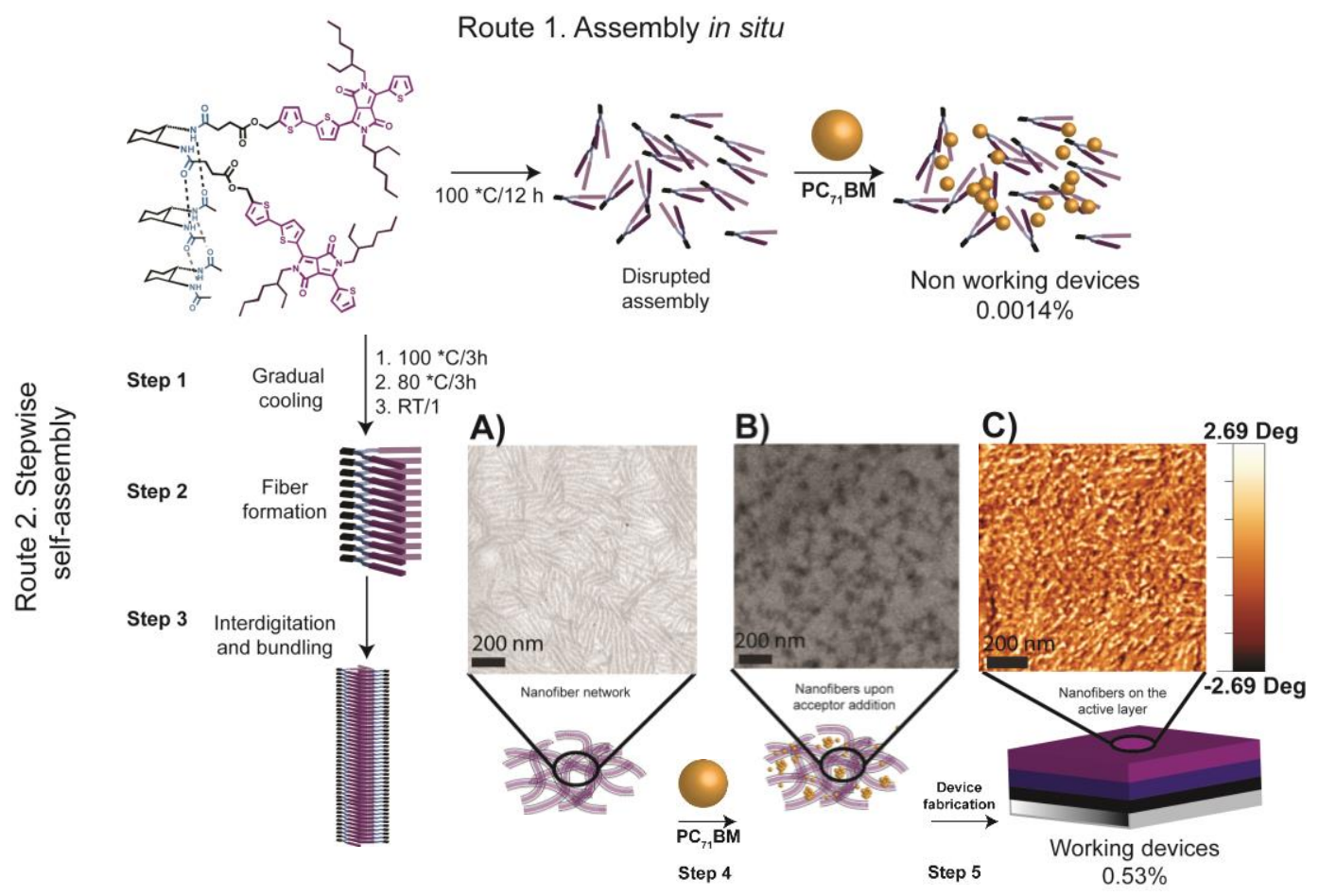

Figure 12. Schematic representation of the preparation pathways used for device fabrication. Pathway 1 (top) shows the single step self-assembly of DPPPHP and $\mathbf{P C}_{71} \mathbf{B M}$. Route 2 (bottom) shows the stepwise selfassembly of DPPHP prior to the addition of $\mathbf{P C}_{71} \mathbf{B M}$. Cryo-TEM image of a DPPHP solution in toluene prepared following the stepwise self-assembly pathway. [DPPHP] $=7 \mathrm{mg} \mathrm{ml}^{-1}(\mathbf{A})$. Cryo-TEM image of a DPPHP:PC $\mathbf{F 1}_{71} \mathbf{B M}$ blend in a $1: 1$ ratio mixed for 30 seconds. [DPPHP] $=7 \mathrm{mg} \mathrm{ml}^{-1}$ and $\left[\mathbf{P C}_{71} \mathbf{B M}\right]=27 \mathrm{mg}$ $\mathrm{ml}^{-1}$ (B). AFM image of the active layer of a device fabricated by the stepwise self-assembly pathway (C).

Reproduced from Ref. 92 with permission from The Royal Society of Chemistry.

Despite the proof-of-concept strategy used by Stupp et al., ${ }^{101}$ the solar cell efficiency obtained was too low. Therefore, the same group proposed the use of linear H-bonded DPPs (A-Amide) containing terminal amide groups (Figure 13) for the fabrication of BHJSCs. In this case, symmetric and asymmetric H-bonded DPP derivatives were studied, finding that the asymmetric derivatives showed improved solubility and more efficient devices. The authors synthesized control molecules containing ester functional groups instead of amides (A-Ester). Both, A-Amide and A-Ester, showed very similar optoelectronic properties but different morphologies on thin film. While A-Ester active layers revealed 
greater crystallinity and $\pi$ - $\pi$ stacking analyzed by grazing incidence X-ray diffraction (GIXD), the active layers of devices made with A-Amide presented short fiber-like supramolecular aggregates with much smaller domain sizes and less ordered than the A-Ester active layers. Interestingly, the devices fabricated in blends with $\mathbf{P C} \mathbf{C}_{71} \mathbf{B M}$ show PCE 50\% higher for A-Amide than for A-Ester. All the results point out that the H-bonds compete with long-range $\pi-\pi$ stacking interactions, resulting in interconnected and nanoscale smaller donor domains vs highly crystalline larger domains obtained with A-Ester. Such morphology seems to nearly double the device efficiency when the H-bonded donors are present. The authors showed that with a good compromise between solubility and the formation of the appropriate nanostructures, this general strategy could be used to optimize function. Remarkably, there were simultaneous studies reported by other groups where H-bonded semiconductors different to DPP were functionalized with terminal amides. In this case, the presence of H-bonding resulted to be detrimental for device performance. ${ }^{102}$ However, only one solvent was used to prepare the devices and it is possible that poor solubility played an important role in that case.

The terminal amide strategy has been reported by other groups, finding very interesting results and applications. For example, Ghosh et al. ${ }^{103}$ reported a DPP-based small molecule (DPP-Amide) with terminal amide groups that formed a black colored gel in toluene (Figure 14a and 14b). In this case, the derivative reported was symmetric, containing dodecyl branches as solubilizing alkyl tails, vs butyloctyl alkyl tails in the previous example. The details on the gel formation and properties will be discussed below in the gels section (Section 5). Figure 14c shows the absorption spectra of DPP-Amide in monomeric state using chloroform as a good solvent and in aggregated state in toluene. It can be observed that the spectrum in aggregated state is very different from the spectrum in the molecularly dissolved state, appearing typical bands corresponding to the formation of $\mathrm{H}$ - and $\mathrm{J}$-aggregates, a behavior previously observed in other $\mathbf{D P P}{ }^{104,105}$ and H-bonded systems. ${ }^{106}$ 


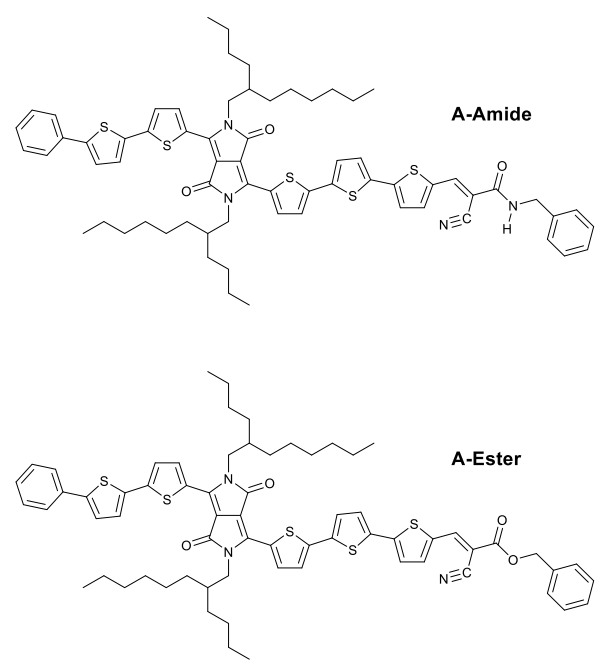

(a)

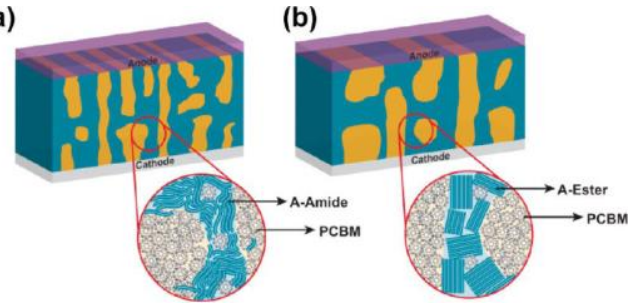

Figure 13. Structures of A-Amide and A-Ester and schematic illustration of active layer morphology for A-Amide (a) and A-Ester devices (b). Adapted with permission from "T. Aytun, L. Barreda, A. RuizCarretero, J. A. Lehrman and S. I. Stupp, Chem. Mater., 2015, 27, 1201-1209”. Copyright 2015, American Chemical Society.
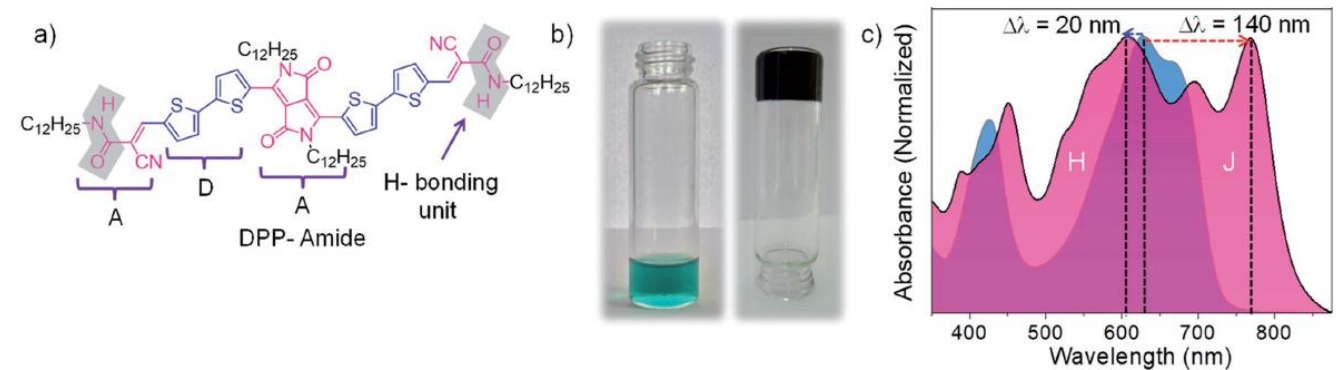

Figure 14. a) Molecular structure of DPP-Amide. b) Photograph of a dilute solution of DPP-Amide in chloroform and the gel formed in toluene (inverted vial). c) UV-Vis spectra of DPP-Amide in monomeric state and aggregated state. Adapted with permission from "S. Ghosh, S. Cherumukkil, C. H. Suresh and A. Ajayaghosh, Adv. Mater., 2017, 29, 1703783”. Copyright (2017). Jon Wiley and Sons.

DPP-Amide was successfully employed to fabricate a composite material together with poly(PDMS). A small amount of the self-assembled fibers formed by DPP-Amide were incorporated into the 
PDMS matrix, obtaining a self-standing filter. Thanks to its absorption properties, covering the visible region and being transparent in the NIR region could be employed in anti-counterfeiting, infrared photography and forensic applications. Furthermore, DPP-Amide was employed to fabricate a hybrid system with $\mathbf{P C}_{71} \mathbf{B M}$, resulting in the formation of a hybrid gel under certain conditions (Figure 15). ${ }^{107}$ In this case, a self-sorted p-n heterojunctions with broad absorption and low band, which was not possible with other hybrid systems especially the ones based on perylene bisimides. ${ }^{108}$ Further details on this hybrid systems are given in the section dedicated to DPP hydrogen-bonded gelators (vide infra). The importance of the self-assembled state by H-bonding was observed by measuring emission quenching upon the addition of $\mathbf{P C} \mathbf{C}_{71} \mathbf{B M}$. While the addition of $\mathbf{P C} \mathbf{C}_{71} \mathbf{B M}$ to solutions of DPP-Amide or DPP-Ester in their molecularly dissolved state (chloroform) no emission quenching was observed, indicating the absence of electron transfer processes, finding the same effect in toluene solutions of DPP-Ester. In contrast, when different ratios of $\mathbf{P C} \mathbf{C}_{71} \mathbf{B M}$ were added to toluene solutions of DPP-Amide (aggregated conditions), attenuation in the emission signal of DPP-Amide was observed with increasing weight ratio of $\mathbf{P C}_{71} \mathbf{B M}$ (Figura 15b). Such attenuation was not due to ground state charge transfer since the absorption spectrum of the DPP-Amide: $\mathbf{P C} \mathbf{C}_{71} \mathbf{B M}$ mixture did not show any additional bands, being the sum of the spectral features of both components (Figure 15a). These results indicate that the selfassembled structures of DPP-Amide are necessary to immobilize $\mathbf{P C} \mathbf{C}_{\mathbf{7 1}} \mathbf{B M}$ aggregates and to facilitate the electron transfer process. Flash-photolysis time resolved microwave conductivity (FP-TRMC) was used to study charge carrier mobility and photovoltaic performance, which can be predicted by plotting transient photoconductivity vs different blending ratios of acceptor material. FP-TRMC measurements were performed on assembled (toluene) and disassembled samples (chloroform) of DPP-Amide using different ratios of $\mathbf{P C}_{\mathbf{7 1}} \mathbf{B M}$, finding a 5-fold increase in intrinsic conductivity for self-assembled films of DPP-Amide:PC ${ }_{71} \mathbf{B M}$ in 1.5:1-1:1.5 ratio (Figure 15c). No enhancement in conductivity was observed in DPP-Ester: $\mathbf{P C}_{71} \mathbf{B M}$ films. 

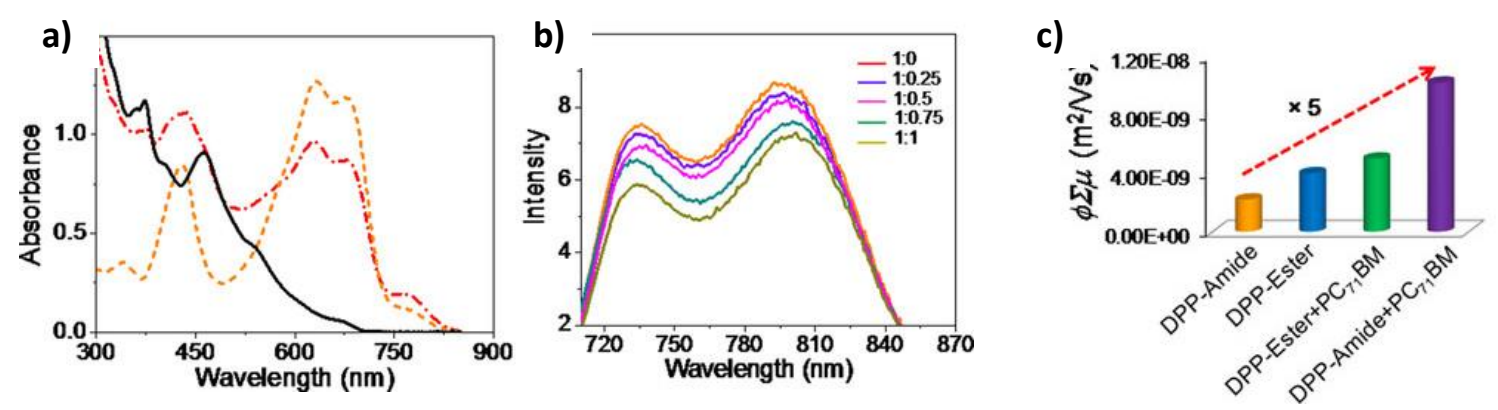

Figure 15. a) Absorption spectra of DPP-Amide aggregates (orange, dash), $\mathbf{P C} \mathbf{7 1}_{\mathbf{1}} \mathbf{B M}$ (black, solid) and DPP-Amide : $\mathbf{P C} \mathbf{7 1}_{71} \mathbf{B M}(1: 1$ wt. ratio) hybrid assembly (red, dash-dot). b) Emission spectra of DPP-

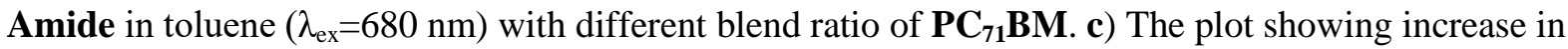
photoconductivity of DPP-Amide and DPP-Ester upon addition of $\mathbf{P C}_{71} \mathbf{B M}$ (1: 1 wt. ratio). Adapted with permission from "S. Ghosh, S. Das, A. Saeki, V. K. Praveen, S. Seki and A. Ajayaghosh, ChemNanoMat, 2018, 4, 831-836”. Copyright (2018). Jon Wiley and Sons.

More recently, the terminal functionalization of DPP semiconductors has been used to modulate charge carrier polarity. Ghosh et al., ${ }^{109}$ reported three DPP derivatives with the same $\pi$-conjugated backbone symmetrically functionalized with amide, ester and cyano groups (Figure 16). The authors used FP-TRMC as well to confirm that the n-type charge carrier mobility increased with higher acceptor strength and OFET devices were fabricated to confirm the change of polarity from p- to n-type. The photoconductivity of the three derivatives on thin film was measured either in their pristine state or by mixing them with $\mathbf{P C}_{71} \mathbf{B M}$ to analyze the p-type charge mobility. When the amide-functionalized derivative was spin-coated from toluene solutions, an enhancement in photoconductivity was observed in comparison to the chloroform films. This was a clear indication of the importance of the H-bonding formation. Interestingly, in blends with $\mathbf{P C}_{71} \mathbf{B M}$ (Figure 17b) the amide-functionalized derivative showed enhanced photoconductivity related to the pristine film. On the other hand, the blends made with the 
ester-functionalized DPP exhibited much lower photoconductivity enhancement with respect to the pristine film and a decrease in photoconductivity in blends made with the dicyano derivative.
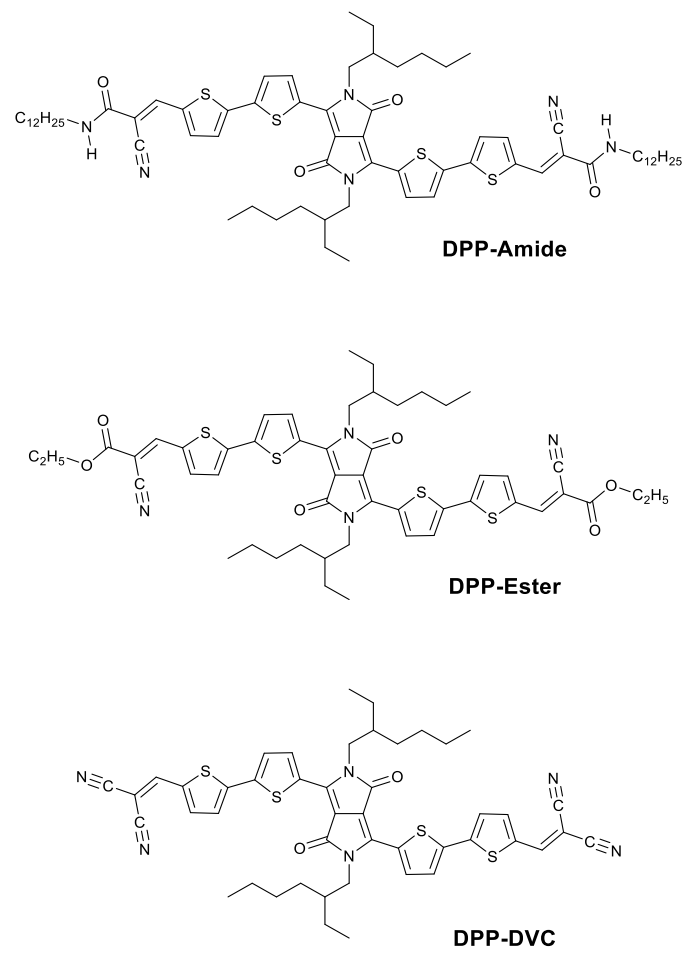

Figure 16. Structures of the three diphenyl-DPP derivatives used to modulate charger carrier polarity. Adapted with permissions from "S. Ghosh, R. Raveendran, A. Saeki, S. Seki, M. Namboothiry and A. Ajayaghosh, ACS Appl. Mater. Interfaces, 2019, 11, 1088-1095" Copyrights 2019, American Chemical Society.

These results indicate a transition from p-type character of the amide derivative to n-type character in the cyano derivative. Inversely, the three derivatives were blended with poly (3-hexylthiophene) (P3HT) (Figure $17 \mathrm{a}, \mathrm{b}, \mathrm{c}$ ) to evaluate the n-type character, finding in this case the opposite trend. However, the solvent used in this case was chlorobenzene instead of chloroform, which could yield different supramolecular structures with different electronic properties, especially in the case of the H-bonded 
derivative. The mobility values were calculated by fabricating FET devices, finding $\mu_{\mathrm{h}}$ of $1.3 \times 10^{-2} \mathrm{~cm}^{2}$ $\mathrm{V}^{-1} \mathrm{~s}^{-1}$ for the amide derivative, while $\mu_{\mathrm{e}}$ of $1.5 \times 10^{-2} \mathrm{~cm}^{2} \mathrm{~V}^{-1} \mathrm{~s}^{-1}$ for the ester derivative and of $1 \times 10^{-2}$ $\mathrm{cm}^{2} \mathrm{~V}^{-1} \mathrm{~s}^{-1}$ for the dicyano DPP. With these results the authors offered an alternative strategy to modulate charge transport properties.
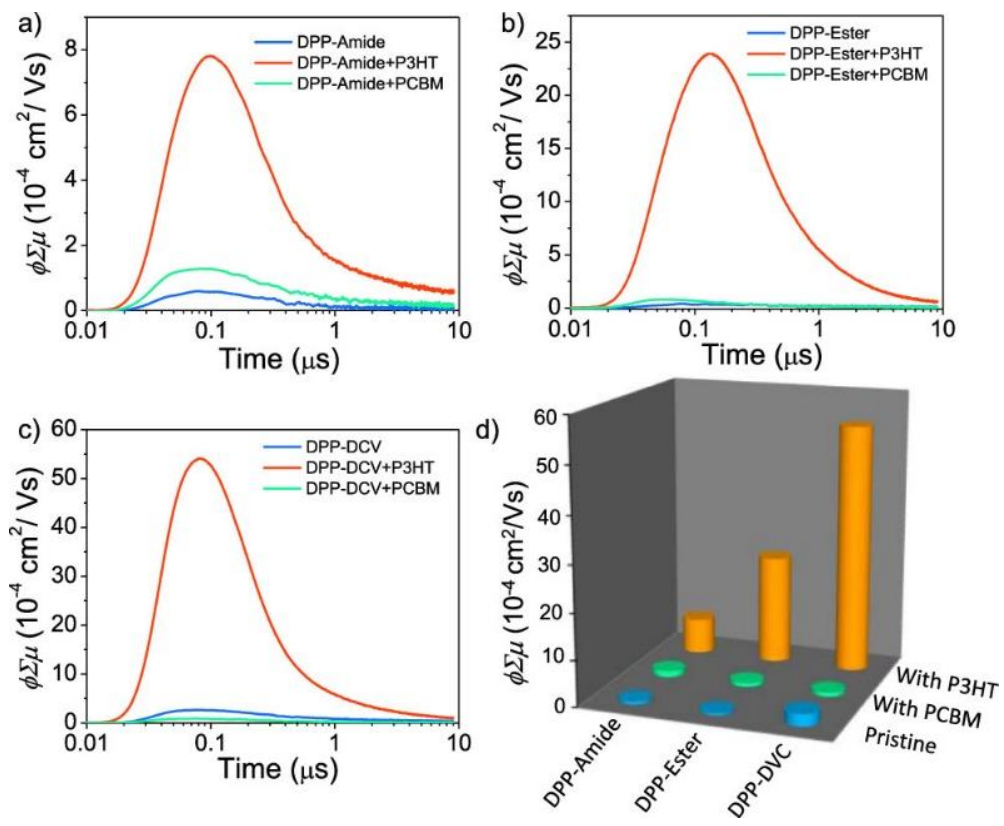

Figure 17. FP-TRMC transients of a) DPP-amide, DPP- amide:P3HT, and DPP-amide:PC ${ }_{71} \mathbf{B M}$; b) DPP-ester, DPP- ester:P3HT, and DPP-ester:PC ${ }_{71} \mathbf{B M}$; and c) DPP-DCV, DPP-DCV:P3HT, and DPP-DCV:PC ${ }_{71}$ BM. d) Comparison of $\phi \Sigma \mu$ values of all the DPP derivatives in the absence and presence of P3HT and $\mathbf{P C}_{71} \mathbf{B M}$. Reprinted with permissions from "S. Ghosh, R. Raveendran, A. Saeki, S. Seki, M. Namboothiry and A. Ajayaghosh, ACS Appl. Mater. Interfaces, 2019, 11, 1088-1095”. Copyrights 2019, American Chemical Society.

\subsection{Complementary H-bonding groups incorporated in DPP derivatives}

Complementary H-bonding between two conjugated species to form heteroaggregates is a great strategy to achieve hierarchical structures, being p-n heterojunctions in the case of semiconductors. In this 
sense, H-bonding motifs able to form multiple and complementary bonds with other conjugated systems have been incorporated into DPP derivatives. Braunschweig et al., ${ }^{110,111}$ studied systems composed of DPP donors with chiral and achiral side chains. These systems can form triple H-bonds with perylene diimide (PDI) acceptors and assemble into superstructures (Figure 18a, b, c). They discovered that by heating solutions containing both type of molecules, achiral PDIs bind to disordered DPP stacks upon cooling, this then leads to the development of chiral superstructures. Additionally, a model was developed to elucidate the structural cues that induce the transition from a disordered aggregate into a chiral helix. The major breakthrough of this model is that it allowed the quantitative determination of all the thermodynamic parameters, while also establishing that the H-bonding and the subsequent helix formation process are enthalpically favored but entropically disfavored. ${ }^{110}$ Recently, this same group has explored six superstructures featuring different geometries, resulting in subtle changes in the solid-state packing of the DPPs. ${ }^{112}$ They achieved this by performing combinations of two DPPs and three rylenes to form six hierarchical superstructures that assemble as a result of orthogonal $\mathrm{H}$-bonding and $\pi \cdots \pi$ stacking (Figure 18d, e, f). The changes in inter-DPP stacking that are templated by the neighboring rylenes have a subtle effect on the excited-state dynamics and on the activation of new pathways such as singlet fission (SF). The exclusive benefits of combinatorial supramolecular assembly demonstrated the impact of the structure on advanced light management in the form of SF, affording triplet quantum yields as high as $65 \%$ for a correlated pair of triplets and $15 \%$ for an uncorrelated pair of triplets. ${ }^{112}$ The changes in the molecular structures of the components resulted in various $1 \mathrm{D}$ and $2 \mathrm{D}$ morphologies, whose long-range order and geometric differences have an influence on the management of light. Further studies of these supramolecular scaffoldings will be important to understand the morphology dependence on the photophysics of the supramolecular heterojunctions in order to maximize the junction area to achieve efficient charge separation and transport. ${ }^{112}$ 
a)

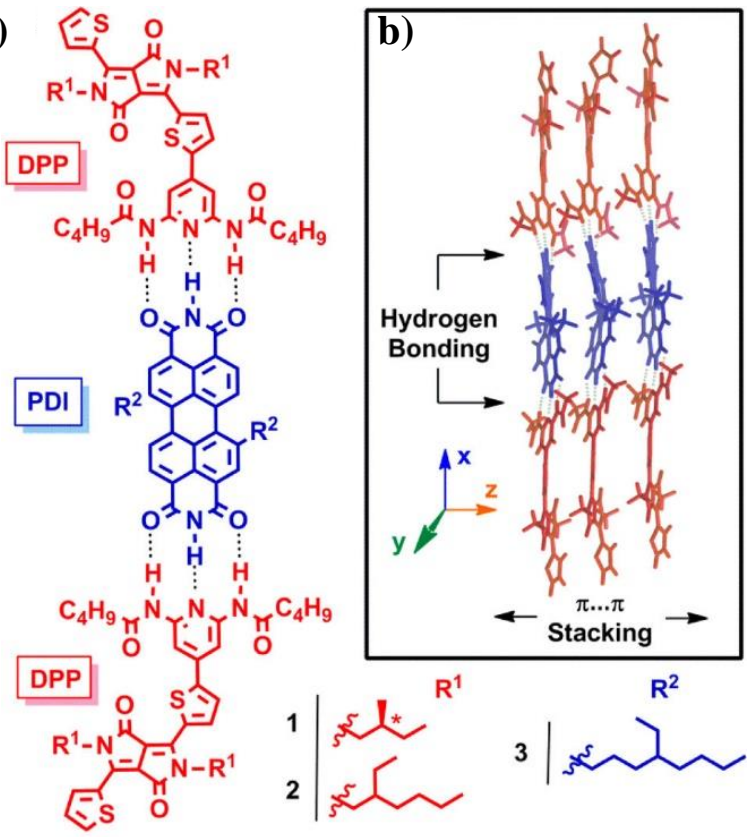

c)

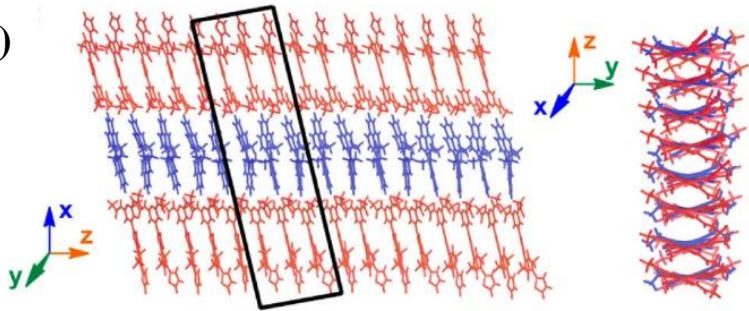

d)

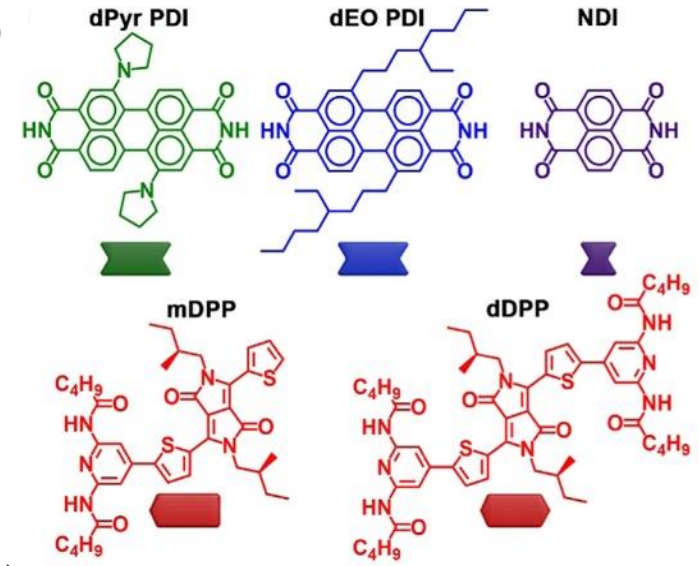

e)

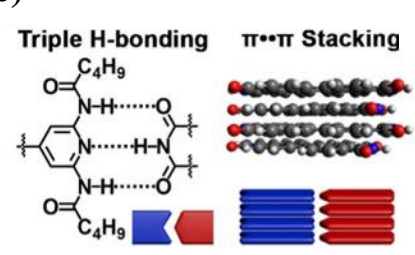

Superstructure Formation

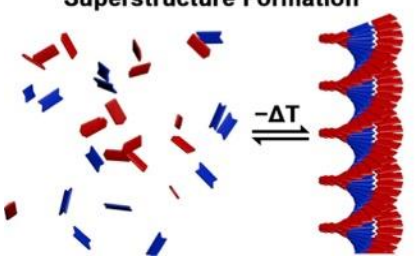

f)

IDPP-dPyr PDI (1:1) aCD $]_{n} \Sigma$ mDPP-dPyr PDI (2:1) dDPP-dEO PDI (1:1) Cप $\sum \prod_{n} \Sigma$ MDPP-DEO PDI (2:1) dDPP-NDI (1:1) $\Sigma[\square \square]_{n} \Sigma$ mDPP-NDI (2:1)

Figure 18. a) DPP donor (red) and PDI acceptor (blue) molecules 1-3 are capable of b) heteroaggregation through a combination of H-bonding and $\pi \cdots \pi$ stacking, resulting in c) well-ordered superstructures. Adapted with permission from "S. Rieth, Z. Li, C. E. Hinkle, C. X. Guzman, J. J. Lee, S. I. Nehme and A. B. Braunschweig, J. Phys. Chem. C, 2013, 117, 11347-11356”. Copyright 2013, American Chemical Society. Supramolecular library, assembly mechanism, and combinatorial systems. d) Library composed of three rylenes and two diketopyrrolopyrroles, e) supramolecular assembly via triple H-bonding and $\pi \cdots \pi$ stacking results in DPP-rylene superstructures, and f)superstructures vary in the DPP and rylene components and DPP/rylene stoichiometry. Adapted with permission from "A. M. Levine, C. Schierl, B. S. Basel, M. Ahmed, B. A. Camargo, D. M. Guldi and A. B. Braunschweig, J. Phys. Chem. C, 2019, 123, 1587-1595”. Copyright 2019, American Chemical Society. 
Braunschweig et al., have also performed studies to determine how solubilizing side chains, the conjugation length and the type of H-bonding groups affect the homo-assembly of DPPs into Jaggregates. ${ }^{113}$ These studies were performed by variable temperature (VT) UV-Vis titrations in toluene on multiple thiophene DPP derivatives (Figure 19). They reported that the presence of diamidopyridine (DAP) groups and their ability to form H-bonds improved considerably the driving force for assembly into larger clusters compared to those without the DAP group. According to their findings, the interplay of $\pi \cdots \pi$ stacking, H-bonding, van der Waals forces, solvent, concentration and temperature is responsible for the size, structure, stability and spectroscopic attributes of the resulting superstructures. Moreover, the main discovery in this work was that these molecules assemble into slip-stacked geometries, described as J-aggregates. They also managed to derive two equations for the calculation of the size of aggregated stacks and its average mole fraction. ${ }^{113}$ These findings are very important since the structure and properties of supramolecular assemblies are intimately linked, and small changes in the relative orientations of stacks can have great impact on their optoelectronic properties and device performance. ${ }^{114,115}$
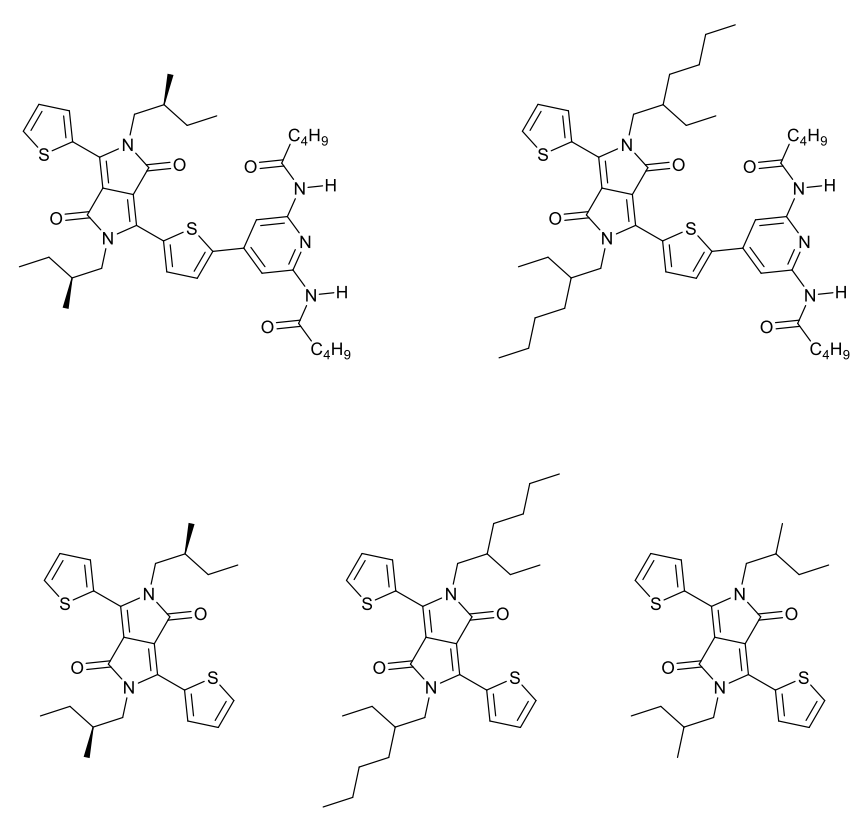

Figure 19. DPP derivatives whose aggregation was studied by Braunschweig et al. ${ }^{116}$ 


\subsection{Other H-bonding motifs present in DPP derivatives}

Our group has shown for example the introduction of semicarbazone functionalities into the simple thiophene-capped DPP core. ${ }^{117}$ Two DPP derivatives were synthesized (Figure 20a and 20b) and the appearance of J-aggregates due to H-bonding formation was observed. We observed the increase of the Jaggregate band in solvents that promote the formation of H-bonds $v s$ chloroform, which was used as a good solvent. The addition of methanol, a solvent that competes with the formation of H-bonds, resulted in the disappearance of the J-aggregate bands, putting in evidence the participation of H-bonds in the formation of such kinds of aggregates (Figure 20c and d). Furthermore, by tuning the aggregation state of these simple derivatives using different solvents, concentrations and temperature, it was possible to tune the energy bandgaps and cover large regions of the solar spectrum even reaching the NIR region. Different 1D structures were found, which presented different optical properties, making these small molecules very interesting to compare the different aggregation conditions on device efficiency.

a)

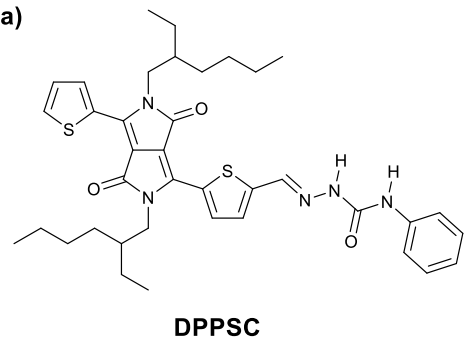

c)

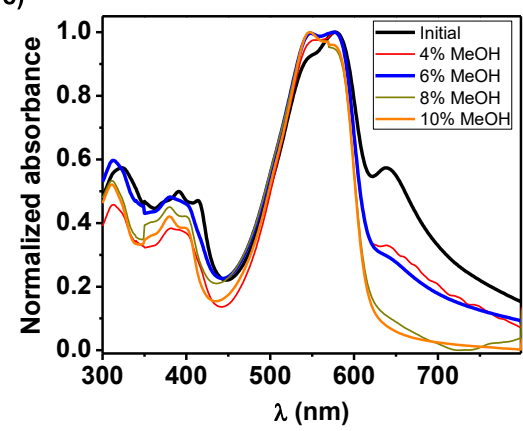

b)

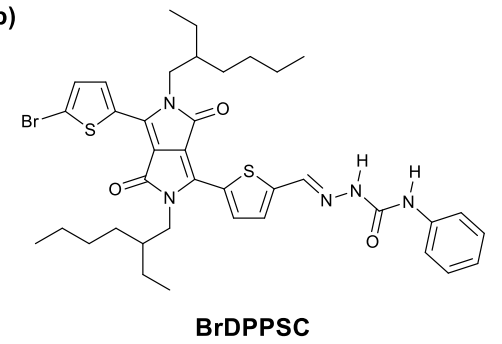

d)

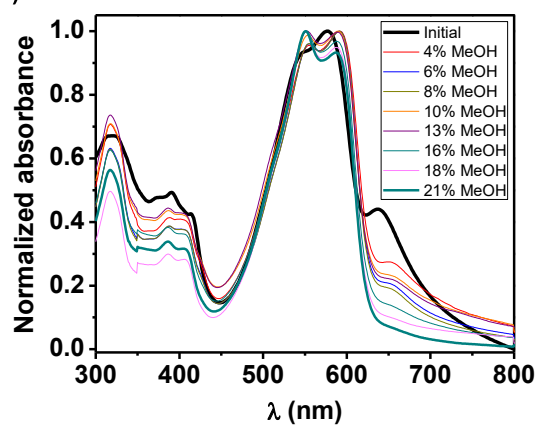

Figure 20. Structures of DPPSC (a) and Br DPPSC (b). Normalized absorption spectra in solution of DPPSC (c) and (d) BrDPPC upon the addition of methanol. 
The presence of semicarbazone groups has also been found in other DPP derivatives that were used as colorimetric chemosensors for fluoride ions. ${ }^{10}$ This same group reported another chemosensor for fluoride ions using phenylhydrazone ${ }^{11}$ as the H-bonding unit. In these cases, the H-bonding groups were employed to bind the fluoride ions and the supramolecular structures formed by them were not studied.

Reynolds et al, ${ }^{118}$ have shown DPP derivatives containing triglyme chains (DPP ${ }_{\text {amphi }}$, Figure 21a) to provide amphiphilic character to the molecule and to achieve solubility and control on the solid state morphology. The self-assembly process of $\mathbf{D P P}_{\text {amphi }}$ were studied by UV-Vis spectroscopy (Figure 21c), probing the absorption at different times, the evolution of the absorption bands and the appearance of new bands $(750 \mathrm{~nm})$ show the formation of aggregates. The mobility values obtained with transistors fabricated with this molecule were not higher than the values reported at the time, but the processing and purification processes were easier due to the desirable solubility. Furthermore, the fill factor $(F F)$ values obtained in BHJSCs were higher than the $F F$ reported in devices at that time. An example of a bolaamphiphilic DPP derivative has also been reported ${ }^{119}$ to obtain supramolecular nanofibers by combination of H-bonding and $\pi$ - $\pi$ stacking (Figure 21b). Two carboxylic acids were attached to the DPP core through a $\mathrm{C} 10$ alkyl linker to obtain the bola-amphiphile compound that resulted to form nanofibers in aqueous solution. The self-assembly properties of DPP-11a were studied by UV-Vis spectroscopy in different solvents, using tetrahydrofuran (THF) as a good solvent and water as a bad solvent and probing the absorption spectra at different times (Figure 21d). In this case, as well as in the case of $\mathbf{D P P} \mathbf{P}_{\text {amphi }}$, the evolution of the bands and the appearance of aggregate bands (J-aggregate) manifest the important role of H-bonds to tune the formation of different morphologies. In this case, THF solutions drop cast on mica showed the formation of nanoparticles, while the aqueous solutions presented the formation of nanofibers. 
a)

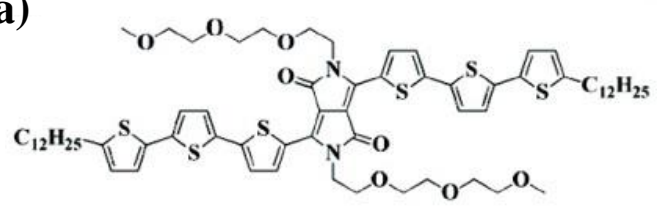

c)

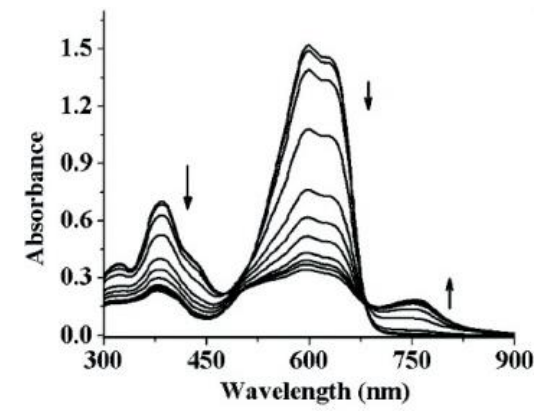

b)

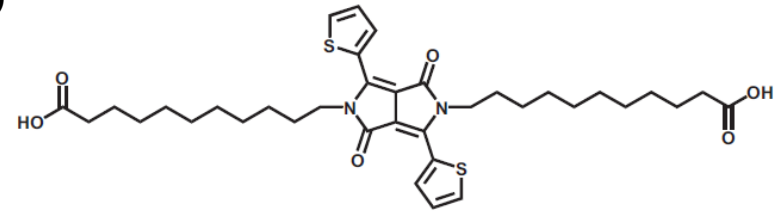

d)

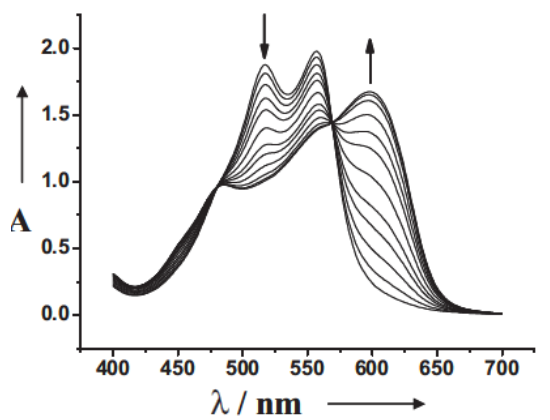

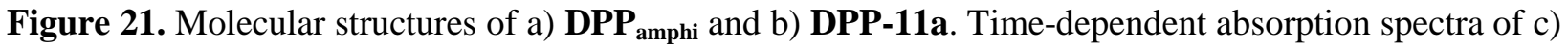
DPP $_{\text {amphi }}$ and d) DPP-11a. a) and c) Adapted with permission from (J. Mei, K. R. Graham, R. Stalder, S. P. Tiwari, H. Cheun, J. Shim, M. Yoshio, C. Nuckolls, B. Kippelen, R. K. Castellano and J. R. Reynolds, Chem. Mater., 2011, 23, 2285-2288.). Copyright (2011) American Chemical Society. b) and d) Adapted with permission from (B. Song, H. Wei, Z. Wang, X. Zhang, M. Smet and W. Dehaen, Adv. Mater., 2007, 19, 416-420). Copyright (2007) Jon Wiley and Sons.

\subsection{Hydrogen bonds incorporated into DPP-based polymers}

H-bonding groups have also been integrated into the structure of semiconducting polymers to achieve enhanced charge transport and expand the applications into sensing devices. Zhang et al ${ }^{120}$ incorporated thymine groups in the side chains of a thiophene-functionalized DPP polymer (PDPP4T-6) (Figure 22a). Thymine is one of the four nucleobases in DNA and has been demonstrated to bind with certain metal ions, such as $\mathrm{Pd}(\mathrm{II})$ and $\mathrm{Hg}(\mathrm{II}) .{ }^{121}$ FET devices were fabricated by spin-coating solutions of DPP4T-T and controls molecule PDPP4T-A (with branched alkyl tails) and PDPP4T-B (with branched and linear alkyl tails), finding $\mu_{\mathrm{h}}$ values higher for the polymer containing thymine. This result was rationalized 
taking into account the enhancement of the film crystallinity due to H-bonding in comparison with the conjugated polymers containing only alkyl chains. This was demonstrated with grazing incidence wideangle X-ray scattering (GIWAXS) measurements, which showed improved lamellar stacking than the films made with the control molecules. Furthermore, the FET devices made with the thymine-containing polymer were used as sensors for $\mathrm{CO}$ and $\mathrm{H}_{2} \mathrm{~S}$. The incorporation of $\mathrm{Pd}(\mathrm{II})$ and $\mathrm{Hg}(\mathrm{II})$ was achieved through the air-water interface coordination. After the metallic ion coordination, FETs were fabricated and exposed to different gaseous analytes and solvent vapours. The polymer containing Pd(II) could detect $\mathrm{CO}$ in concentrations as low as $10 \mathrm{ppb}$, while devices made with $\mathrm{Hg}$ (II) coordinated ions could sense $\mathrm{S}_{2} \mathrm{Hg}$ down to $1 \mathrm{ppb}$. In this case, the introduction of H-bonding functionalities in semiconducting polymers resulted not only in a mobility enhancement, but also in additional applications and properties.

Urea groups have also been introduced in the pending chains of DPP polymers. Zhang et al, ${ }^{122}$ reported three DPP-based polymers containing different ratios of urea groups and branched alkyl chains (Figure 22b). OFETS and BHJSCs were fabricated with these polymers and the control DPP polymer without urea units. The authors found out that the polymer containing the lowest ratio (1:30) of urea groups, showed the highest hole mobility $\left(\mu_{\mathrm{h}}=13.1 \mathrm{~cm}^{2} \mathrm{~V}^{-1} \mathrm{~s}^{-1}\right)$. The influence of the presence of urea groups in DPP polymers was also studied in BHJSCs, finding in this case that the polymer with the highest ratio of urea groups (1:30) presented the best efficiency of all (6.8\%), including the control devices made with polymers without urea groups. The high mobility values found were attributed to the enhancement of lamellar packing order of the alkyl said chains on the thin films prepared thanks to the $\mathrm{H}$ bonding interactions led by the urea groups and the appearance of inter-chain $\pi-\pi$ stacking after thermal annealing at $100{ }^{\circ} \mathrm{C}$. Regarding the solar cell devices, the presence of urea groups was found to guide the assembly of the polymers into nanofibers and direct the ordered aggregation of the acceptor material, resulting in micro-phase separation in the blends were charge separation can be enhanced, compared to the control polymers without urea groups. 

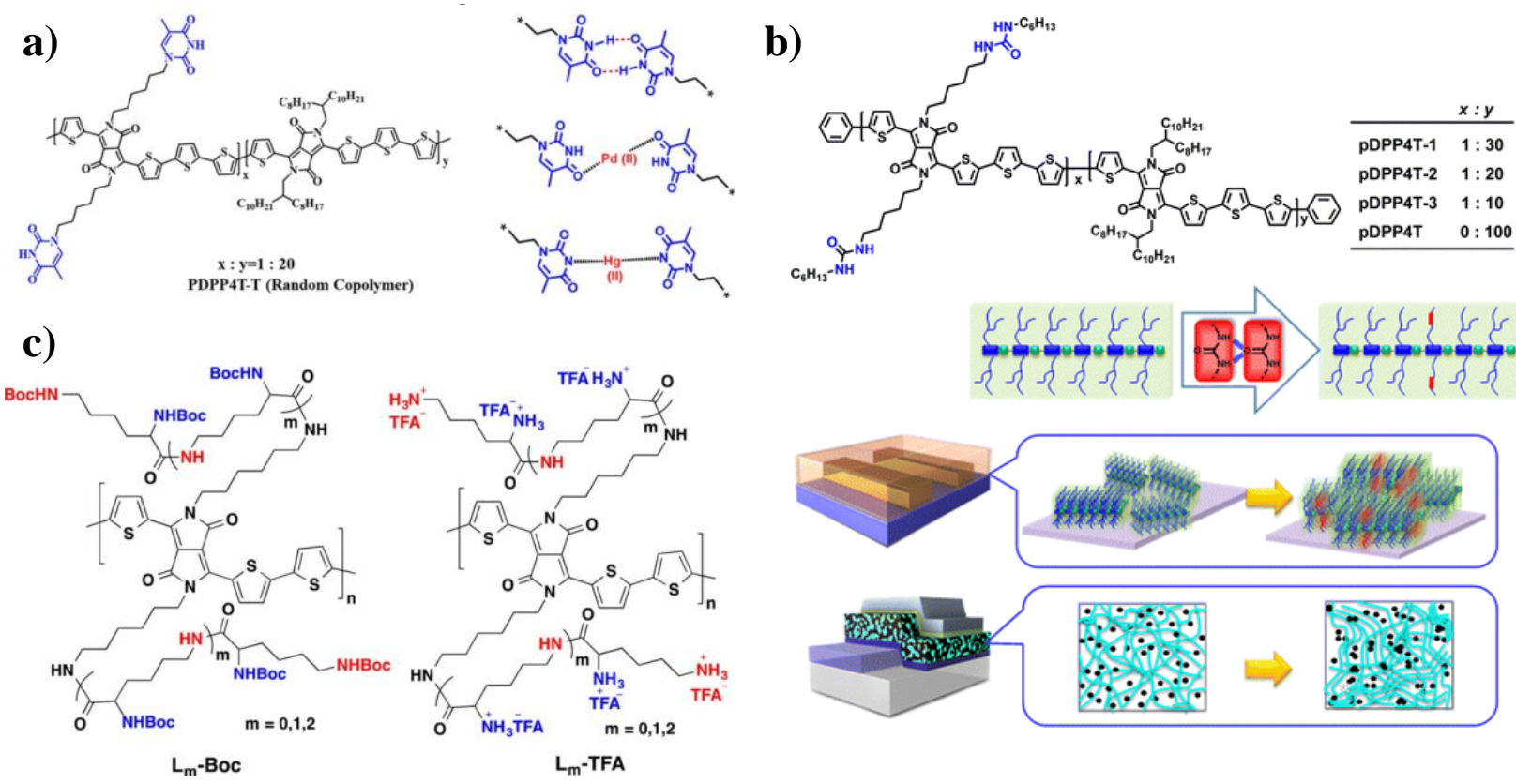

Figure 22. a) Chemical Structure of PDPP4T-T; Thymine groups are randomly connected to the conjugated backbone and the illustration of $\mathrm{T}-\mathrm{T}$ Intermolecular $\mathrm{H}$-bonding and the coordination with Pd(II) and Hg(II). Reprinted with permission from "Y. Yang, Z. Liu, L. Chen, J. Yao, G. Lin, X. Zhang, G. Zhang and D. Zhang, Chem. Mater., 2019, 31, 1800-1807”. Copyright 2019, American Chemical Society. b) Chemical Structures of pDPP4T-1, pDPP4T-2, pDPP4T-3, and pDPP4T, and illustration of the design rationale for the incorporation of urea groups in the side chains of conjugated $\mathrm{D}-\mathrm{A}$ polymers. Reprinted with permission from "J. Yao, C. Yu, Z. Liu, H. Luo, Y. Yang, G. Zhang and D. Zhang, J. Am. Chem. Soc., 2016, 138, 173-185”. Copyright 2016, American Chemical Society. c) Reprinted with permission from (W. Du, D. Ohayon, C. Combe, L. Mottier, I. P. Maria, R. S. Ashraf, H. Fiumelli, S. Inal and I. McCulloch, Chem. Mater., 2018, 30, 6164-6172). Copyright (2018) American Chemical Society.

The use of H-bonds in DPP polymers has also interesting applications in bioelectronics. Du et al, ${ }^{123}$ reported a DPP polymer containing lysine side chains (Figure 22c) that was applied in neural cell adhesion and growth. This way, it was not necessary to use other biological coating steps on electrical 
devices, making them promising materials for applications in bioelectronics. DPP polymers based on DPP3T (dithienyl-DPP) containing different number of lysine derivatives (Figure 22c) were synthesized. However, the strong H-bonding ability of lysine resulted in very strong intermolecular aggregation and lack of solubility. The lysine groups were protected with $t$-Boc groups to provide solubility and deprotected subsequently with trifluroacetic acid (TFA) to render protonated lysine units, which increased the surface charge and improved cell adhesion.

\section{Organogels and hydrogels made with hydrogen-bonded DPP derivatives}

\subsection{Organogels based on DPP}

The preparation of organogels and hydrogels is an attractive strategy for developing complex supramolecular structures. In the recent years, the highly ordered and flexible supramolecular architectures found in gels have been applied in organic electronic devices. ${ }^{124}$ Many of these gels are obtained thanks to the presence of noncovalent interactions into the molecular structure of the components. Therefore, the presence of one or multiple H-bonding groups can lead to the formation of gels that in combination with other noncovalent forces, such as $\pi-\pi$ stacking result in great flexible semiconducting materials. Among the organo- and hydrogels reported in literature, only a few examples based on H-bonded DPP can be found. Thool et al. ${ }^{125}$ reported in 2016 the ability of small H-bonded DPP molecules to form organogels. They designed a DPP small molecule containing two phenyl rings at the 3- and 6- positions of the DPP core (Figure 23), which self-assembled using Van der Waals interactions thanks to the presence of saturated carbon chains, $\pi-\pi$ stacking of the aromatic rings and $\mathrm{H}$ bonding groups attached at the periphery phenyl groups. Gel-like materials were obtained by a classical heat/cool method using different solvents in short times, at $0.5 \mathrm{wt} \%$ concentration. Despite the inverted test tube was performed, no complementary rheology studies were shown. The self-assembly process was followed with UV-Vis spectroscopy and microscopy (AFM and HR-TEM). The thin film and gel spectra show red-shifted absorption band and the presence of J-aggregates originated by H-bonding interactions. Particularly, the gel spectrum onset presents a red-shift of more than $100 \mathrm{~nm}$, enlarging the coverage of 
the solar spectrum with respect to the samples in dilute solution. Rod-shaped assemblies with high aspect ratio were found in spin-coated films made with the organogel, which are desirable structures in organic electronics. Organic solar cells were prepared using a known conjugated polymer (PTB7) ${ }^{126}$ as the donor molecule and $\mathbf{P C} \mathbf{7 1}_{\mathbf{7 1}} \mathbf{B M}$ as the acceptor, introducing a small amount of the H-bonded DPP derivative as an additive, either in the neat or gel state. Remarkably, the results show that the device efficiency increases from $6.37 \%$ in devices without additive up to $7.23 \%$ when DPP-NCO is used in the neat state as an additive, and to $7.85 \%$ when the additive is incorporated in the gel state. The authors argue the enhancement in efficiency is due to the formation of a nanorods network in the blend's active layer that decreases the series resistance and improves the $F F$.
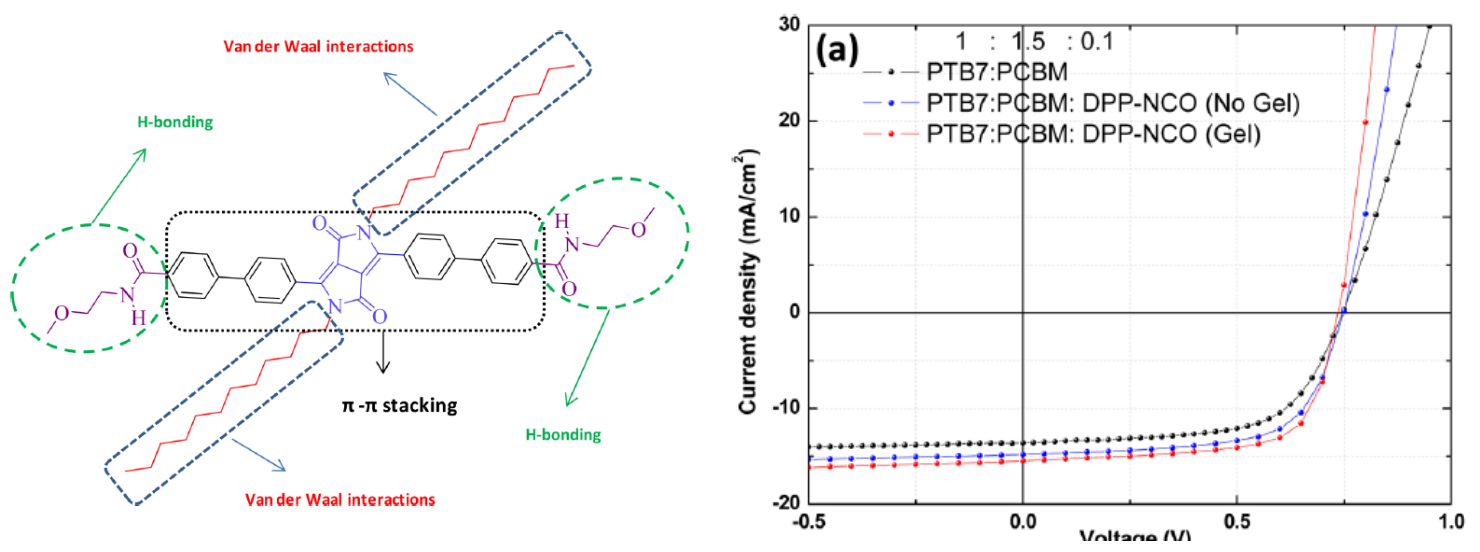

Figure 23. a) Molecular structure of DPP-NCO. b) Organogel of DPP-NCO made in dichloromethane. (c) J-V curves of the devices fabricated with PTBT7:PC ${ }_{71}$ BM:DPP-NCO in 1:1.5:0.1 ratio. Reprinted with permission from (S. Thool, K. Narayanaswamy, A. Venkateswararao, S. Naqvi, V. Gupta, S. Chand, V. Vivekananthan, R. R. Koner, V. Krishnan and S. P. Singh, Langmuir, 2016, 32, 4346-4351). Copyright (2016) American Chemical Society.

Ghosh et al. ${ }^{103}$ reported a new DPP small based molecule with terminal H-bonding groups which formed a black colored gel in toluene (Figure 14). The optical properties of DPP-Amide in the 
aggregated state has been described in the previous section, finding the presence of $\mathrm{H}$ - and J-type aggregates very important for the desired applications. DPP-Amide was found to form gels in toluene at $0.15 \mathrm{wt} \%$ concentration. SEM images of the xerogel show a network of nanofibers that are bundled resulting in large fibers with diameters between $400-500 \mathrm{~nm}$. As explained in the previous section, these fibers were used to fabricate the NIR transparent filter by entrapping them in a PDMS film. X-ray scattering techniques were used to confirm that the structure and properties of the DPP fibers were not affected by their incorporation into the composite. As also seen previously, DPP-Amide was blended with $\mathbf{P C}_{71} \mathbf{B M}$ to fabricate hybrid organogels able to form a coaxial $\mathrm{p}$-n heterojuction, where only selfassembled systems based on H-bonded DPP showed increased conductivity. In this case, no rheological characterization or gelation mechanisms are shown, but a gel-like material was still obtained upon the addition of $\mathbf{P C}_{71} \mathbf{B M}$ to an aggregated sample of DPP-Amide.

Apart from the terminal functionalization of DPP derivatives with H-bonding groups, DPP organogels have been reported using $\mathrm{H}$-bonding units attached to polymers. Rondeu-Gagné et al. ${ }^{127}$ reported the design of a DPP gelator having amide and diacetylene moieties, which could form organogels in several solvents. The authors offered a new strategy to achieve more rigidity and stability in supramolecular systems and gels. Three different gelators were synthesized (Figure 24a), one containing H-bonding groups (G1), another one having diacetylene moieties to rigidify the network through photopolymerization (G2) and G3 containing both groups. Interestingly, only G3 containing amide and diacetylene moieties resulted to form robust gels in aromatic solvents. Upon UV irradiation (Figure 24b) the xerogel of $\mathbf{G 3}$ underwent a topochemical polymerization to form polydiacetylene, resulting in a crosslinked network maintaining the initial fibrous morphology of the gels made by G3. In this case, the authors found out that the H-bond units were critical to obtain the necessary structure for the dyine moieties to polymerize. This strategy is a way to achieve easy and efficient crosslinking without the use of a catalyst or photoinitiators, which is very important for the processing of $\pi$-conjugated materials in thin film devices. This way, a gel could be used as an initial material and be rigidified afterwards to lock the structure. 


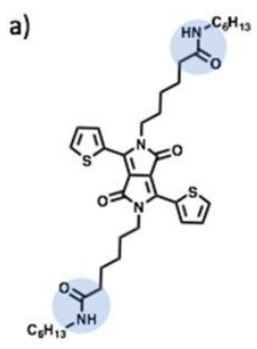

G1

b)

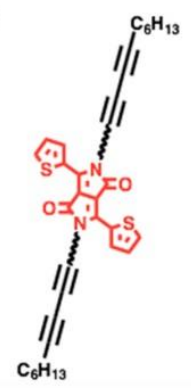

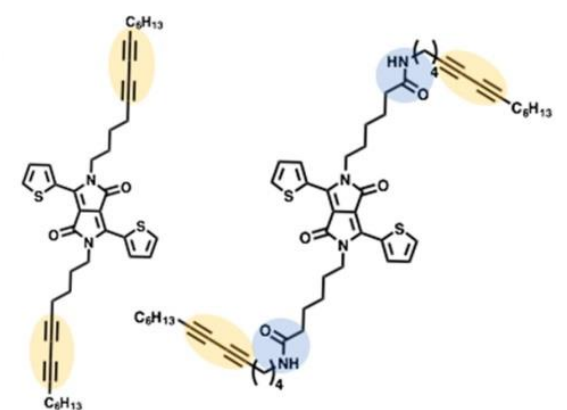

G2

G3

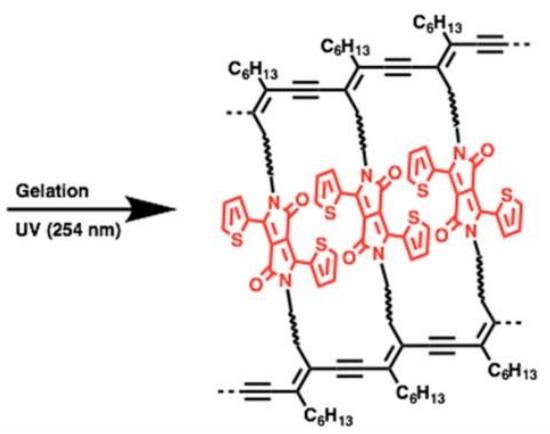

Figure 24. a) Molecular structures of gelators G1, G2 and G3. b) Gelation and cross-linking mechanism. Reprinted with permission from (A. Nyayachavadi, G. T. Mason, M. Nazir Tahir, M. U. Ocheje and S. Rondeau-Gagné, Langmuir, 2018, 34, 12126-12136). Copyright (2018) American Chemical Society.

\subsection{Hydrogels based on DPP}

Draper et al. ${ }^{128}$ reported the first hydrogel formed by a DPP-based low molecular weight gelator (LMWG) (DPP-1) for which gelation was triggered by pH decrease (Figure 25). DPP-1 contains two Lphenylalanine aminoacids pending from the lactam nitrogen. This way, the carboxylic acids of the phenylalanines can be used to solubilize DPP-1 at high $\mathrm{pH}$ and drive self-assembly upon protonation. Gels were prepared by dissolving DPP-1 in an aqueous $0.1 \mathrm{M} \mathrm{NaOH}$ solution reaching $\mathrm{pH}=8$. The decrease of $\mathrm{pH}(\mathrm{pH}=3.3)$ was triggered by adding glucono- $\delta$-lactone $(\mathrm{GdL})$, resulting in slow hydrolysis and the formation of a gel (Figure x). Rheology analysis showed the typical behavior of a LMWG, with gelation starting at $\mathrm{pH}=7.3$ and 8 minutes after GdL was added. DPP-1 was co-assembled with a perylene-based n-type LMWG in an attempt to achieve a p-n heterojunction through self-sorting of both 
gelators. Rheology, $\mathrm{pH}$ and NMR studies show that the two gelators assemble at different times, and the $\mathrm{UV}-\mathrm{Vis}$ absorption spectrum of the co-assembled system differs from the spectra of the individual xerogel was lower than the current of the n-type xerogel alone, probably due to inappropriate band-gap overlap between both components and possible recombination originated from intimately mixed gelator fibers. Nonetheless, a different n-type gelator with appropriate energy levels could be combined with DPP-1, resulting in conductive systems processed from water, a cheaper and more environmentally friendly solvent.

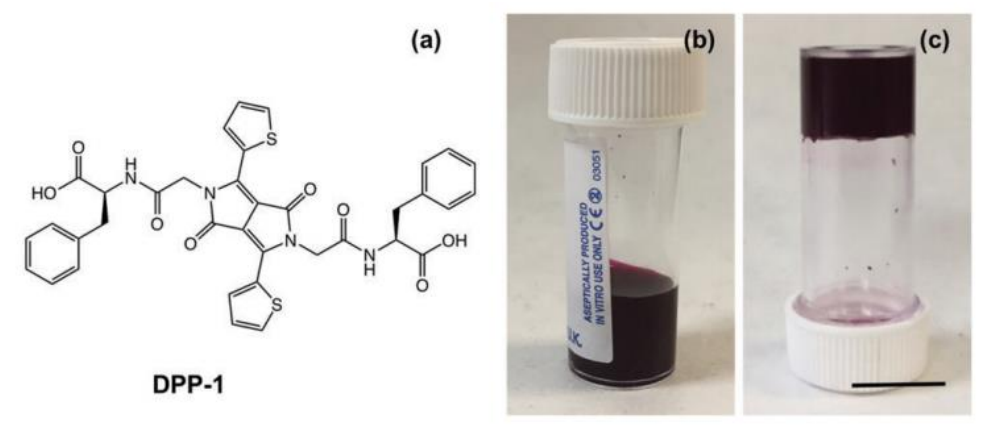

Figure 25. a) Molecular structure of DPP-1. b) Photograph of a solution of DPP-1at pH 8 and a concentration of $5 \mathrm{mg} \mathrm{mL}^{-1}$. c) Photograph of a gel of DPP-1 at pH 3.3 formed by lowering the $\mathrm{pH}$ of the solution shown in (b). Reprinted from "E. R. Draper, B. Dietrich and D. J. Adams, Chem Commun, 2017, 53, 1864-1867" published by The Royal Society of Chemistry.

\section{Conclusions and perspectives}

The field of organic electronics keeps continuously on the search of new materials and approaches to improve device performance. However, the race for achieving record efficiency values and high performance hinders the study of other strategies that can efficiently contribute to the field. This is the case of the use of H-bonds incorporated into the molecular structure of organic semiconductors. The power of this strategy has been outlined in this review, focusing the attention of DPP dyes due to their great potential for functionalization and outstanding optoelectronic properties. Not only H-bonds arising from the amide groups in DPP can be employed, but its versatile functionalization with multiple $\mathrm{H}$ - 
bonding motifs can guide the self-assembly processes of these systems into highly organized structures with optimal properties. Furthermore, it is possible to tune the optoelectronic properties and morphology by varying the aggregation state through H-bonding. Still many efforts need to be done in this field and more particularly, using DPP as the electroactive segment. Systematic studies are necessary to clarify the impact of this strategy, including all possible parameters that can have an influence. The search for n-type hydrogen-bonded DPP materials that can be processed from solution or gel state are among the next topics to be pursued, together with the study of supramolecular chirality.

Finally, focus should be made on the use of greener solvents to study self-assembly in H-bonded DPP derivatives and device fabrication. This way, a sustainable and environmentally friendly global approach towards energy-related issues could be pursued.

\section{References}

1 J.-M. Lehn, Angew. Chem. Int. Ed. Engl., 1990, 29, 1304-1319.

2 D. González-Rodríguez and A. P. H. J. Schenning, Chem. Mater., 2011, 23, 310-325.

3 T. Aida, E. W. Meijer and S. I. Stupp, Science, 2012, 335, 813-817.

4 A. D. Hendsbee, J.-P. Sun, L. R. Rutledge, I. G. Hill and G. C. Welch, J. Mater. Chem. A, 2014, 2, 4198-4207.

5 E. D. Głowacki, H. Coskun, M. A. Blood-Forsythe, U. Monkowius, L. Leonat, M. Grzybowski, D. Gryko, M. S.

White, A. Aspuru-Guzik and N. S. Sariciftci, Org. Electron., 2014, 15, 3521-3528.

6 D. G. Farnum, G. Mehta, G. G. I. Moore and F. P. Siegal, Tetrahedron Lett., 1974, 15, 2549-2552.

7 W. Li, K. H. Hendriks, M. M. Wienk and R. A. J. Janssen, Acc. Chem. Res., 2016, 49, 78-85.

8 J. C. Bijleveld, A. P. Zoombelt, S. G. . Mathijssen, M. M. Wienk, M. Turbiez, D. M. de Leeuw and R. A. . Janssen, J. Am. Chem. Soc., 2009, 131, 16616-16617.

9 M. Stolte, S.-L. Suraru, P. Diemer, T. He, C. Burschka, U. Zschieschang, H. Klauk and F. Würthner, Adv. Funct. Mater., 2016, 26, 7415-7422.

10 X. Yang, L. Zheng, L. Xie, Z. Liu, Y. Li, R. Ning, G. Zhang, X. Gong, B. Gao, C. Liu, Y. Cui, G. Sun and G. Zhang, Sens. Actuators B Chem., 2015, 207, 9-24.

11 X. Yang, L. Xie, R. Ning, X. Gong, Z. Liu, Y. Li, L. Zheng, G. Zhang, B. Gao, Y. Cui, G. Sun and G. Zhang, Sens. Actuators B Chem., 2015, 210, 784-794.

12 H. Bürckstümmer, A. Weissenstein, D. Bialas and F. Würthner, J. Org. Chem., 2011, 76, 2426-2432.

13 C. J. . Morton, R. Gilmour, D. M. Smith, P. Lightfoot, A. M. . Slawin and E. J. MacLean, Tetrahedron, 2002, 58, 5547-5565.

14 D. Feng, G. Barton and C. N. Scott, Org. Lett., 2019, 21, 1973-1978.

15 D. Ranganathan, Further Challenging Problems in Organic Reaction Mechanisms, Elsevier, 2012.

16 United States, US4579949A, 1986.

17 United States, US4613669A, 1986.

18 E. B. Faulkner and R. J. Schwartz, Eds., High Performance Pigments, Wiley-VCH Verlag GmbH \& Co. KGaA, Weinheim, Germany, 2009.

19 Number of patents based on Google Patents database (https://www.google.com/patents), 2019.

20 A. Tang, C. Zhan, J. Yao and E. Zhou, Adv. Mater., 2017, 29, 1600013.

21 B. Lim, H. Sun, J. Lee and Y.-Y. Noh, Sci. Rep., 2017, 7, 164.

22 O. Ostroverkhova, Chem. Rev., 2016, 116, 13279-13412. 
24 M. Sassi, N. Buccheri, M. Rooney, C. Botta, F. Bruni, U. Giovanella, S. Brovelli and L. Beverina, Sci. Rep., 2016, 6, 34096.

25 D. Chandran and K.-S. Lee, Macromol. Res., 2013, 21, 272-283.

26 M. Grzybowski and D. T. Gryko, Adv. Opt. Mater., 2015, 3, 280-320.

27 A. Iqbal, M. Jost, R. Kirchmayr, J. Pfenninger, A. Rochat and O. Wallquist, Bull. Sociétés Chim. Belg., 2010, 97, 615-644.

28 M. Kaur and D. H. Choi, Chem. Soc. Rev., 2015, 44, 58-77.

29 M. Grzybowski, E. Glodkowska-Mrowka, G. Clermont, M. Blanchard-Desce and D. T. Gryko, Chem. Heterocycl. Compd., 2017, 53, 72-77.

30 A. Chiminazzo, G. Borsato, A. Favero, C. Fabbro, C. E. McKenna, L. G. Dalle Carbonare, M. T. Valenti, F. Fabris and A. Scarso, Chem. - Eur. J., 2019, 25, 3617-3626.

31 T. Potrawa and H. Langhals, Chem. Ber., 1987, 120, 1075-1078.

32 F. Nourmohammadian and S. Shiva Shamekhi, Lett. Org. Chem., 2013, 10, 131-138.

33 S. S. Shamekhi and F. Nourmohammadian, Pigment Resin Technol., 2013, 42, $215-222$.

34 A. Shaabani, M. Dabiri, A. Bazgir and K. Gharanjig, Dyes Pigments, 2006, 71, 68-72.

35 Y. Ji, C. Xiao, Q. Wang, J. Zhang, C. Li, Y. Wu, Z. Wei, X. Zhan, W. Hu, Z. Wang, R. A. J. Janssen and W. Li, Adv. Mater., 2016, 28, 943-950.

36 T. Aysha, S. Luňák, A. Lyčka, J. Vyňuchal, Z. Eliáš, A. Růžička, Z. Padělková and R. Hrdina, Dyes Pigments, 2013, 98, 530-539.

37 S. Ding, Z. Ni, M. Hu, G. Qiu, J. Li, J. Ye, X. Zhang, F. Liu, H. Dong and W. Hu, Macromol. Rapid Commun., 2018, 39, 1800225.

38 G. Qiu, Z. Jiang, Z. Ni, H. Wang, H. Dong, J. Zhang, X. Zhang, Z. Shu, K. Lu, Y. Zhen, Z. Wei and W. Hu, J. Mater. Chem. C, 2017, 5, 566-572.

39 C. J. H. Morton, R. L. Riggs, D. M. Smith, N. J. Westwood, P. Lightfoot and A. M. Z. Slawin, Tetrahedron, 2005, 61, 727-738.

40 R. L. Riggs, C. J. H. Morton, A. M. Z. Slawin, D. M. Smith, N. J. Westwood, W. S. D. Austen and K. E. Stuart, Tetrahedron, 2005, 61, 11230-11243.

$41 \quad$ United States, US7442804B2, 2008.

$42 \quad$ World Intellectual Property Organization, WO2003022848A3, 2003.

43 F. Closs and R. Gompper, Angew. Chem. Int. Ed. Engl., 1987, 26, 552-554.

44 F. Closs, R. Gompper, H. Nöth and H.-U. Wagner, Angew. Chem. Int. Ed. Engl., 1988, 27, 842-845.

45 J. Z. Zabicky, The Chemistry of Amides, Interscience Publishers, 1970.

46 F. Pop, J. Humphreys, J. Schwarz, L. Brown, A. van den Berg and D. B. Amabilino, New J. Chem., , DOI:10.1039/C9NJ01074B.

47 S. A. Shevelev, Russ. Chem. Rev., 1970, 39, 844-858.

48 M. Grzybowski and D. T. Gryko, Adv. Opt. Mater., 2015, 3, 280-320.

49 S. Stas, S. Sergeyev and Y. Geerts, Tetrahedron, 2010, 66, 1837-1845.

50 Š. Frebort, Z. Eliáš, A. Lyčka, S. Luňák, J. Vyňuchal, L. Kubáč, R. Hrdina and L. Burgert, Tetrahedron Lett., 2011, 52, 5769-5773.

51 S. Stas, J.-Y. Balandier, V. Lemaur, O. Fenwick, G. Tregnago, F. Quist, F. Cacialli, J. Cornil and Y. H. Geerts, Dyes Pigments, 2013, 97, 198-208.

$52 \quad$ United States, US4914211A, 1990.

53 C. H. Woo, P. M. Beaujuge, T. W. Holcombe, O. P. Lee and J. M. J. Fréchet, J. Am. Chem. Soc., 2010, 132, 15547-15549.

54 A. B. Tamayo, M. Tantiwiwat, B. Walker and T.-Q. Nguyen, J. Phys. Chem. C, 2008, 112, $15543-15552$.

55 L. Dou, W.-H. Chang, J. Gao, C.-C. Chen, J. You and Y. Yang, Adv. Mater., 2013, 25, 825-831.

56 G. P. McGlacken and L. M. Bateman, Chem. Soc. Rev., 2009, 38, 2447.

57 W. Han and A. Ofial, Synlett, 2011, 2011, 1951-1955.

58 Y. Li, P. Sonar, L. Murphy and W. Hong, Energy Environ. Sci., 2013, 6, 1684-.

59 S. Qu and H. Tian, Chem. Commun., 2012, 48, 3039.

60 B. P. Karsten, J. C. Bijleveld and R. A. J. Janssen, Macromol. Rapid Commun., 2010, 31, $1554-1559$.

61 W. Hong, B. Sun, H. Aziz, W.-T. Park, Y.-Y. Noh and Y. Li, Chem. Commun., 2012, 48, 8413.

62 J. Mizuguchi, J Phys Chem A, 2000, 104, 1817-1821.

63 Z. Hao and A. Iqbal, Chem. Soc. Rev., 1997, 26, 203-213.

64 G. R. Desiraju, J. Mol. Struct., 2003, 656, 5-15. 
65 M. Irimia-Vladu, E. D. Głowacki, P. A. Troshin, G. Schwabegger, L. Leonat, D. K. Susarova, O. Krystal, M. Ullah, Y. Kanbur, M. A. Bodea, V. F. Razumov, H. Sitter, S. Bauer and N. S. Sariciftci, Adv. Mater., 2012, 24, 375-380.

66 E. D. Gllowacki, G. Voss, L. Leonat, M. Irimia-Vladu, S. Bauer and N. S. Sariciftci, Isr. J. Chem., 2012, $\mathbf{5 2}, 540-551$.

67 D. Basak, D. S. Pal, T. Sakurai, S. Yoneda, S. Seki and S. Ghosh, Phys. Chem. Chem. Phys., 2017, 19, 31024-31029.

68 E. D. Głowacki, M. Irimia-Vladu, M. Kaltenbrunner, J. Gsiorowski, M. S. White, U. Monkowius, G.

Romanazzi, G. P. Suranna, P. Mastrorilli, T. Sekitani, S. Bauer, T. Someya, L. Torsi and N. S. Sariciftci, Adv.

Mater., 2013, 25, 1563-1569.

69 H. Yanagisawa, J. Mizuguchi, S. Aramaki and Y. Sakai, Jpn. J. Appl. Phys., 2008, 47, 4728.

70 E. D. Głowacki, H. Coskun, M. A. Blood-Forsythe, U. Monkowius, L. Leonat, M. Grzybowski, D. Gryko,

M. S. White, A. Aspuru-Guzik and N. S. Sariciftci, Org. Electron., 2014, 15, 3521-3528.

71 A. Facchetti, Chem. Mater., 2011, 23, 733-758.

72 J. Mizuguchi and G. Wooden, Berichte Bunsenges. Für Phys. Chem., 1991, 95, 1264-1274.

73 J. Mizuguchi and G. Rihs, Berichte Bunsenges. Für Phys. Chem., 1992, 96, 597-606.

74 J. Mizuguchi, A. Grubenmann, G. Wooden and G. Rihs, Acta Crystallogr. B, 1992, 48, 696-700.

75 J. Mizuguchi, A. Grubenmann and G. Rihs, Acta Crystallogr. B, 1993, 49, 1056-1060.

76 J. Mizuguchi, G. Giller and E. Baeriswyl, J. Appl. Phys., 1994, 75, 514-518.

77 J. Mizuguchi, A. Grubenmann, G. Wooden and G. Rihs, Acta Crystallogr. B, 1992, 48, 696-700.

78 J. Mizuguchi, J. Appl. Phys., 1989, 66, 3111-3113.

79 J. Mizuguchi and S. Homma, J. Appl. Phys., 1989, 66, 3104-3110.

80 J. Mizuguchi, A. C. Rochat and G. Rihs, Berichte Bunsenges. Für Phys. Chem., 1992, 96, 607-619.

81 J. Mizuguchi, Berichte Bunsenges. Für Phys. Chem., 1993, 97, 684-693.

82 J. Mizuguchi, T. Imoda, H. Takahashi and H. Yamakami, Dyes Pigments, 2006, 68, 47-52.

83 C. Fu, P. J. Beldon and D. F. Perepichka, Chem. Mater., 2017, 29, 2979-2987.

84 J. S. Zambounis, Z. Hao and A. Iqbal, Nature, 1997, 388, 131.

85 S. T. Salammal, J.-Y. Balandier, S. Kumar, E. Goormaghtigh and Y. H. Geerts, Cryst. Growth Des., 2014, 14, 339-349.

86 Y. Suna, J. Nishida, Y. Fujisaki and Y. Yamashita, Org. Lett., 2012, 14, 3356-3359.

87 SunaYuki, NishidaJun-ichi, FujisakiYoshihide and YamashitaYoshiro, Chem. Lett., ,

DOI:10.1246/cl.2011.822.

88 E. Daniel Głowacki, M. Irimia-Vladu, S. Bauer and N. Serdar Sariciftci, J. Mater. Chem. B, 2013, 1, 37423753.

89 K. Yang, L. Xiang, Y. F. Huang, R. S. Bhatta, J. Liu, M. Tsige, C. L. Wang, S. Cheng and Y. Zhu, Polymer, 2019, 238-245.

90 S. Mula, T. Han, T. Heiser, P. Lévêque, N. Leclerc, A. P. Srivastava, A. Ruiz-Carretero and G. Ulrich, Chem. - Eur. J., , DOI:10.1002/chem.201900689.

91 B. Sun, W. Hong, H. Aziz and Y. Li, J. Mater. Chem., 2012, 22, 18950-18955.

92 Y. Li, P. Sonar, S. P. Singh, M. S. Soh, M. van Meurs and J. Tan, J. Am. Chem. Soc., 2011, 133, 21982204.

93 J. Lee, A.-R. Han, J. Hong, J. H. Seo, J. H. Oh and C. Yang, Adv. Funct. Mater., 2012, 22, $4128-4138$.

94 F. Bruni, M. Sassi, M. Campione, U. Giovanella, R. Ruffo, S. Luzzati, F. Meinardi, L. Beverina and S.

Brovelli, Adv. Funct. Mater., 2014, 24, 7410-7419.

95 J. Dhar, D. Prasad Karothu and S. Patil, Chem. Commun., 2015, 51, 97-100.

96 F. Pop, W. Lewis and D. B. Amabilino, CrystEng Comm, 2016, 18, 8933-8943.

97 Y.-J. Zhang, X. Wang, Y. Zhou and C.-K. Wang, Chem. Phys. Lett., 2016, 658, 125-129.

98 C. Yang, M. Zheng, Y. Li, B. Zhang, J. Li, L. Bu, W. Liu, M. Sun, H. Zhang, Y. Tao, S. Xue and W. Yang, J. Mater. Chem. A, 2013, 1, 5172-5178.

99 K. Nie, B. Dong, H. Shi, Z. Liu and B. Liang, Sens. Actuators B Chem., 2017, 244, 849-853.

100 A. Ruiz-Carretero, T. Aytun, C. J. Bruns, C. J. Newcomb, W.-W. Tsai and S. I. Stupp, J. Mater. Chem. A, 2013, 1, 11674.

101 T. Aytun, L. Barreda, A. Ruiz-Carretero, J. A. Lehrman and S. I. Stupp, Chem. Mater., 2015, 27, 12011209.

102 Z. Xiao, K. Sun, J. Subbiah, S. Ji, D. J. Jones and W. W. H. Wong, Sci. Rep., , DOI:10.1038/srep05701.

103 S. Ghosh, S. Cherumukkil, C. H. Suresh and A. Ajayaghosh, Adv. Mater., 2017, 29, 1703783. 
104 M. Kirkus, L. Wang, S. Mothy, D. Beljonne, J. Cornil, R. A. J. Janssen and S. C. J. Meskers, J. Phys. Chem. A, 2012, 116, 7927-7936.

105 M. Más-Montoya and R. A. J. Janssen, Adv. Funct. Mater., , DOI:10.1002/adfm.201605779.

106 W.-W. Tsai, I. D. Tevis, A. S. Tayi, H. Cui and S. I. Stupp, J. Phys. Chem. B, 2010, 114, $14778-14786$.

107 S. Ghosh, S. Das, A. Saeki, V. K. Praveen, S. Seki and A. Ajayaghosh, ChemNanoMat, 2018, 4, 831-836.

108 A. Mishra and P. Bäuerle, Angew. Chem. Int. Ed., 2012, 51, 2020-2067.

109 S. Ghosh, R. Raveendran, A. Saeki, S. Seki, M. Namboothiry and A. Ajayaghosh, ACS Appl. Mater. Interfaces, 2019, 11, 1088-1095.

110 S. Rieth, Z. Li, C. E. Hinkle, C. X. Guzman, J. J. Lee, S. I. Nehme and A. B. Braunschweig, J. Phys. Chem. $C, 2013,117,11347-11356$.

111 D. Ley, C. X. Guzman, K. H. Adolfsson, A. M. Scott and A. B. Braunschweig, J. Am. Chem. Soc., 2014, 136, 7809-7812.

112 A. M. Levine, C. Schierl, B. S. Basel, M. Ahmed, B. A. Camargo, D. M. Guldi and A. B. Braunschweig, J. Phys. Chem. C, 2019, 123, 1587-1595.

113 Y. Zhou, C. X. Guzman, L. C. Helguero-Kelley, C. Liu, S. R. Peurifoy, B. Captain and A. B. Braunschweig, J. Phys. Org. Chem., 2016, 29, 689-699.

114 E. H. A. Beckers, S. C. J. Meskers, A. P. H. J. Schenning, Z. Chen, F. Würthner, P. Marsal, D. Beljonne, J. Cornil and R. A. J. Janssen, J. Am. Chem. Soc., 2006, 128, 649-657.

115 R. F. Fink, J. Seibt, V. Engel, M. Renz, M. Kaupp, S. Lochbrunner, H.-M. Zhao, J. Pfister, F. Würthner and B. Engels, J. Am. Chem. Soc., 2008, 130, 12858-12859.

116 Y. Zhou, C. X. Guzman, L. C. Helguero-Kelley, C. Liu, S. R. Peurifoy, B. Captain and A. B. Braunschweig, J. Phys. Org. Chem., 2016, 29, 689-699.

117 S. Militzer, T. M. P. Tran, P. J. Mésini and A. Ruiz-Carretero, ChemNanoMat, 2018, 4, 790-795.

118 J. Mei, K. R. Graham, R. Stalder, S. P. Tiwari, H. Cheun, J. Shim, M. Yoshio, C. Nuckolls, B. Kippelen, R. K. Castellano and J. R. Reynolds, Chem. Mater., 2011, 23, 2285-2288.

119 B. Song, H. Wei, Z. Wang, X. Zhang, M. Smet and W. Dehaen, Adv. Mater., 2007, 19, 416-420.

120 Y. Yang, Z. Liu, L. Chen, J. Yao, G. Lin, X. Zhang, G. Zhang and D. Zhang, Chem. Mater., 2019, 31, 1800-1807.

121 J. Šebera, J. Burda, M. Straka, A. Ono, C. Kojima, Y. Tanaka and V. Sychrovský, Chem. - Eur. J., 2013, 19, 9884-9894.

122 J. Yao, C. Yu, Z. Liu, H. Luo, Y. Yang, G. Zhang and D. Zhang, J. Am. Chem. Soc., 2016, 138, $173-185$.

123 W. Du, D. Ohayon, C. Combe, L. Mottier, I. P. Maria, R. S. Ashraf, H. Fiumelli, S. Inal and I. McCulloch, Chem. Mater., 2018, 30, 6164-6172.

124 S. S. Babu, S. Prasanthkumar and A. Ajayaghosh, Angew. Chem. Int. Ed., 2012, 51, 1766-1776.

125 G. S. Thool, K. Narayanaswamy, A. Venkateswararao, S. Naqvi, V. Gupta, S. Chand, V. Vivekananthan,

R. R. Koner, V. Krishnan and S. P. Singh, Langmuir, 2016, 32, 4346-4351.

126 J. Razzell-Hollis, J. Wade, W. C. Tsoi, Y. Soon, J. Durrant and J.-S. Kim, J. Mater. Chem. A, 2014, 2, 20189-20195.

127 A. Nyayachavadi, G. T. Mason, M. Nazir Tahir, M. U. Ocheje and S. Rondeau-Gagné, Langmuir, 2018, 34, 12126-12136.

128 E. R. Draper, B. Dietrich and D. J. Adams, Chem Commun, 2017, 53, 1864-1867.

\section{Acknowledgments}

A. R. C. and R. A. R. thank the Foundation for Frontier Research in Chemistry (FRC) LabEx Emerging Investigators Grant 2018 and CNRS for financial support. S. M. and P. J. M. thank the International Research Training Center (IRTG) SoMas and the Région Grand-Est for the doctoral fellowship of S. M. 